\title{
THE INTERSTELLAR MEDIUM AND STAR FORMATION IN EDGE-ON GALAXIES. II. NGC 4157, 4565, AND 5907
}

\author{
KiJeong Yim ${ }^{1,2}$, Tony Wong ${ }^{1}$, Rui Xue ${ }^{1}$, Richard J. RANd ${ }^{3}$, Erik Rosolowsky ${ }^{4}$, \\ J. M. van der Hulst ${ }^{2}$, Robert BenJamin ${ }^{5}$, and ERIC J. Murphy ${ }^{6,7}$ \\ ${ }^{1}$ Department of Astronomy, University of Illinois, 1002 West Green Street, Urbana, IL 61801, USA; k.yim@astro.rug.nl \\ ${ }^{2}$ Kapteyn Astronomical Institute, University of Groningen, P.O. Box 800, 9700 AV Groningen, The Netherlands \\ ${ }^{3}$ Department of Physics and Astronomy, University of New Mexico, 1919 Lomas Boulevard NE, Albuquerque, NM 87131-1156, USA \\ ${ }^{4}$ Department of Physics, University of Alberta, CCIS 4-183, Edmonton, AB T6G 2E1, Canada \\ ${ }^{5}$ Department of Physics, University of Wisconsin-Whitewater, 800 West Main Street, Whitewater, WI 53190, USA \\ ${ }^{6}$ Observatories of the Carnegie Institution for Science, 813 Santa Barbara Street, Pasadena, CA 91101, USA \\ ${ }^{7}$ Infrared Processing and Analysis Center, California Institute of Technology, MC 220-6, Pasadena, CA 91125, USA \\ Received 2013 November 8; accepted 2014 August 9; published 2014 November 11
}

\begin{abstract}
We present a study of the vertical structure of the gaseous and stellar disks in a sample of edge-on galaxies (NGC 4157, 4565, and 5907) using BIMA/CARMA ${ }^{12} \mathrm{CO} J=1 \rightarrow 0$, VLA H I, and Spitzer $3.6 \mu \mathrm{m}$ data. In order to take into account projection effects when we measure the disk thickness as a function of radius, we first obtain the inclination by modeling the radio data. Using the measurement of the disk thicknesses and the derived radial profiles of gas and stars, we estimate the corresponding volume densities and vertical velocity dispersions. Both stellar and gas disks have smoothly varying scale heights and velocity dispersions, contrary to assumptions of previous studies. Using the velocity dispersions, we find that the gravitational instability parameter $Q$ follows a fairly uniform profile with radius and is $\geqslant 1$ across the star-forming disk. The star formation law has a slope that is significantly different from those found in more face-on galaxy studies, both in deprojected and pixel-by-pixel plots. Midplane gas pressure based on the varying scale heights and velocity dispersions appears to roughly hold a power-law correlation with the midplane volume density ratio.
\end{abstract}

Key words: galaxies: individual (NGC 4157, NGC 4565, NGC 5907) - galaxies: ISM - galaxies: kinematics and dynamics - stars: formation

Online-only material: color figures

\section{INTRODUCTION}

Our understanding of the relationship between star formation and properties of the interstellar medium (ISM), such as gas content and interstellar pressure, is still rudimentary. Observational studies (e.g., Kennicutt 1998; Wong \& Blitz 2002; Bigiel et al. 2008) have derived a power-law relationship between the star formation rate (SFR) and gas surface density $\left(\Sigma_{\mathrm{SFR}} \propto \Sigma_{\mathrm{gas}}{ }^{n}\right)$, a relationship referred to as the star formation law (SFL) or Kennicutt-Schmidt (K-S) law. Although the relationship is usually expressed in terms of surface density, fundamentally it is the volume density of the gas that should be related to star formation (Schmidt 1959; Ferguson et al. 1998; Krumholz et al. 2012).

In spite of the importance of the volume density, however, most star formation studies have used surface density in combination with an assumed constant value of gas scale height since the disk thickness is usually difficult to measure. The disk thickness is, however, expected to increase with radius for a nearly isothermal disk and such flaring of the gas disk has been measured in recent observations of edge-on galaxies (e.g., O'Brien et al. 2010; Yim et al. 2011). An edgeon galaxy provides the most direct measurement of disk thickness, which for the gas disk enables us to derive the volume density, a quantity that may be better correlated with SFR than surface density.

Elmegreen (1993) derived a theoretical relationship between the molecular gas fraction and the hydrostatic midplane pressure. In this model a high intercloud pressure enhances the formation of $\mathrm{H}_{2}$. Therefore, the pressure is perhaps a critical ingredient for describing star formation, which has been demonstrated to proceed primarily from molecular gas. More recent studies (e.g., Wong \& Blitz 2002; Blitz \& Rosolowsky 2004) have established a strong empirical correlation between the molecular to atomic gas ratio and the hydrostatic midplane pressure. This pressure is inferred by assuming a constant gas velocity dispersion and stellar scale height. However, this assumption may not be valid in general. For example, recent studies (e.g., Tamburro et al. 2009; Yim et al. 2011) have found that the gas velocity dispersion changes with radius. Therefore, it is important to examine the role of the gas pressure in controlling the molecular to atomic gas ratio when radial variations of the velocity dispersions and the scale heights are taken into account. Again, edge-on galaxies provide an important laboratory for this study.

In a previous paper (Yim et al. 2011 hereafter Paper I), we studied the ISM and star formation in the prototypical edge-on galaxy NGC 891. In this second paper, we study the vertical structure of three additional edge-on galaxies (NGC 4157, 4565 , and 5907) to investigate how the ISM properties are related to molecular cloud and star formation. We carry out this study using CO imaging from the Berkeley-Illinois-Maryland Association (BIMA) and the Combined Array for Research in Millimeter-wave Astronomy (CARMA), $\mathrm{H}_{\mathrm{I}}$ imaging from the Very Large Array (VLA), and IR imaging from the Spitzer Space Telescope. We have selected these galaxies primarily based on (1) availability of multi-wavelength data including $\mathrm{CO}, \mathrm{HI}$, and IR $(3.6$ and $24 \mu \mathrm{m})$, (2) high inclination $\left(i>80^{\circ}\right)$, (3) presence of strong $\mathrm{CO}$ emission, and (4) active 
Table 1

Galaxy Properties

\begin{tabular}{|c|c|c|c|c|c|c|c|c|}
\hline $\begin{array}{c}\text { Galaxy } \\
(1)\end{array}$ & $\begin{array}{l}\text { R.A. } \\
\text { (2) }\end{array}$ & $\begin{array}{l}\text { Decl. } \\
(3)\end{array}$ & $\begin{array}{c}D_{25} \\
\left({ }^{\prime}\right) \\
(4)\end{array}$ & $\begin{array}{l}\mathrm{PA} \\
\left({ }^{\circ}\right) \\
(5)\end{array}$ & $\begin{array}{c}\text { Distance } \\
(\mathrm{Mpc}) \\
(6)\end{array}$ & $\begin{array}{c}\text { Inclination } \\
\left({ }^{\circ}\right) \\
(7)\end{array}$ & $\begin{array}{c}V_{\text {sys }} \\
\left(\mathrm{km} \mathrm{s}^{-1}\right) \\
(8)\end{array}$ & $\begin{array}{c}\mathrm{K} \\
\mathrm{mag} \\
(9)\end{array}$ \\
\hline NGC 4157 & 121104.34 & 502906.32 & 6.73 & 63 & 12.9 & 82 & 770 & 7.363 \\
\hline NGC 4565 & 123629.18 & 255527.23 & 16.72 & 135 & 9.7 & 87.5 & 1230 & 6.060 \\
\hline NGC 5907 & 151553.55 & 561943.00 & 12.60 & 115 & 11.0 & 86.5 & 670 & 6.757 \\
\hline
\end{tabular}

Notes. Columns (2) and (3) are the galaxy centers used in this study. Column (4) is the optical diameter in arcminutes and the references for NGC 4157, 4565, and 5907 are Rupen (1991); Verheijen \& Sancisi (2001), and Laine et al. (2010), respectively. Column (5) is the position angle obtained from the $3.6 \mu \mathrm{m}$ image using the MIRIAD task IMFIT. Column (6) is the distance from the literature: Irwin et al. (1999) for NGC 4157, Dahlem et al. (2005) for NGC 4565, and Just et al. (2006) for NGC 5907. Column (7) is the inclination from the literature: Verheijen \& Sancisi (2001) for NGC 4157, Zschaechner et al. (2012) for NGC 4565, and Garcia-Burillo et al. (1997) for NGC 5907. Column (8) is the heliocentric systemic velocity adopted in this paper. Column (9) is the $\mathrm{K}_{s}$-band magnitude from the SIMBAD database.

Table 2

$\mathrm{CO}$ and $\mathrm{Hi}$ Observing Parameters

\begin{tabular}{|c|c|c|c|c|c|c|}
\hline Galaxy & \multicolumn{2}{|c|}{ NGC 4157} & \multicolumn{2}{|c|}{ NGC 4565} & \multicolumn{2}{|c|}{ NGC 5907} \\
\hline Telescope & BIMA & VLA & BIMA \& CARMA & VLA & BIMA \& CARMA & VLA \\
\hline$\theta\left({ }^{\prime \prime}\right)$ & $3.84 \times 3.30$ & $15.69 \times 14.87$ & $3.6 \times 2.95$ & $6.26 \times 5.59$ & $3.48 \times 2.76$ & $15.43 \times 13.84$ \\
\hline$\Delta v\left(\mathrm{~km} \mathrm{~s}^{-1}\right)$ & 10 & 20 & 10 & 20 & 10 & 20 \\
\hline Total flux (Jy km s $\left.{ }^{-1}\right)$ & 1280 & 110 & 1030 & 274 & 1530 & 259 \\
\hline Circular beam & $4^{\prime \prime}(250 \mathrm{pc})$ & $15^{\prime \prime} .7(981 \mathrm{pc})$ & 3.7 (174 pc) & 6."3 (296 pc) & 3.6 (190 pc) & $15.5(821 \mathrm{pc})$ \\
\hline
\end{tabular}

Note. $\theta$ is the angular resolution. $\Delta v$ is the velocity resolution. The original beam has been convolved to a circular beam for the analysis in Section 4.1.

star formation as measured at $60 \mu \mathrm{m}$ by IRAS. The properties of the three galaxies are shown in Table 1. Note that we do not use the inclinations shown in Table 1, but obtain inclinations by modeling in Section 4.1 .

The organization of this paper is as follows. We summarize the observations and the reduction of $\mathrm{CO}, \mathrm{HI}$, and IR data in Section 2 and show the radial distributions of $\Sigma_{\mathrm{H}_{2}}, \Sigma_{\mathrm{HI}}, \Sigma_{*}$, and $\Sigma_{\mathrm{SFR}}$ in Section 3. We measure the inclinations of the less edge-on galaxies (NGC 4157, 4565, and 5907) and scale heights of the gas and stars and derive the vertical velocity dispersions in Section 4. We examine the $\mathrm{K}-\mathrm{S}$ law and the role of the instability parameter $Q$ using our sample of galaxies in Section 5. We investigate and discuss the relationship between the molecular to atomic gas ratio and the interstellar gas pressure when the scale heights and the velocity dispersions vary with radius in Section 6. Finally, our results are summarized in Section 7.

\section{OBSERVATIONS AND DATA REDUCTION}

\subsection{CO Observations}

The BIMA ${ }^{12} \mathrm{CO} J=1 \rightarrow 0$ observations were taken in 2004 toward the edge-on galaxies NGC 4157 (in B, C, and D congurations), NGC 4565 (C and D), and NGC 5907 (C and D) with a five field mosaic oriented along the major axis with a $50^{\prime \prime}$ separation between the primary beam centers. In 2010 and 2011, we observed NGC 4565 and 5907 using CARMA (in C and $\mathrm{D}$ configurations) in order to obtain higher angular resolution. The CARMA observations are mosaics of 11 pointings toward NGC 4565 and 13 pointings toward NGC 5907 with a half beam spacing of $30^{\prime \prime}$. The median baseline lengths are roughly $278 \mathrm{~m}(\mathrm{~B}), 93 \mathrm{~m}(\mathrm{C})$, and $40 \mathrm{~m}(\mathrm{D})$ for
BIMA and $278 \mathrm{~m}$ (C) and $111 \mathrm{~m}$ (D) for CARMA. The instruments and configurations used are summarized in Table 2. All the $\mathrm{CO}$ data were calibrated and imaged using the MIRIAD package. The calibration for each track involved use of MFCAL for bandpass and gain calibration and the BOOTFLUX task for flux calibration. For NGC 4565 and 5907, we combined the separately calibrated BIMA and CARMA data using the MIRIAD task INVERT. All the CO images were produced using natural weighting to achieve the highest possible sensitivity.

The CO integrated intensity maps of NGC 4157,4565 , and 5907 are shown in Figure 1. Throughout this paper, we use $x$ for the coordinate along the major axis (line of nodes), $y$ for the coordinate perpendicular to $x$ in the galaxy plane, and $z$ for the coordinate perpendicular to the galaxy plane. The coordinate along the apparent minor axis is actually a combination of $y$ and $z$ (if not exactly edge-on), so we refer to it as the "minor axis offset." The angular and velocity resolutions, total CO flux in $\mathrm{Jy} \mathrm{km} \mathrm{s}^{-1}$, and the rms noise per channel in $\mathrm{K}$ are presented in Table 2. For the total flux, we used a masked integrated intensity map derived from a gain corrected image cube, limited to the region where the map noise is less than twice its minimum (central) value. The masked map is obtained by blanking regions below a $3 \sigma$ threshold in a smoothed cube. We used a noise-flattened map convolved to $10^{\prime \prime}$ for the smoothed cube, where the noiseflattened map is the original cube divided by the normalized sensitivity image. The average rms noise is obtained from line-free edge channels of the gain corrected cube. Each map is rotated to place the blueshifted side of the disk (lower velocities than $\left.V_{\text {sys }}\right)$ on the negative $x$-axis. 


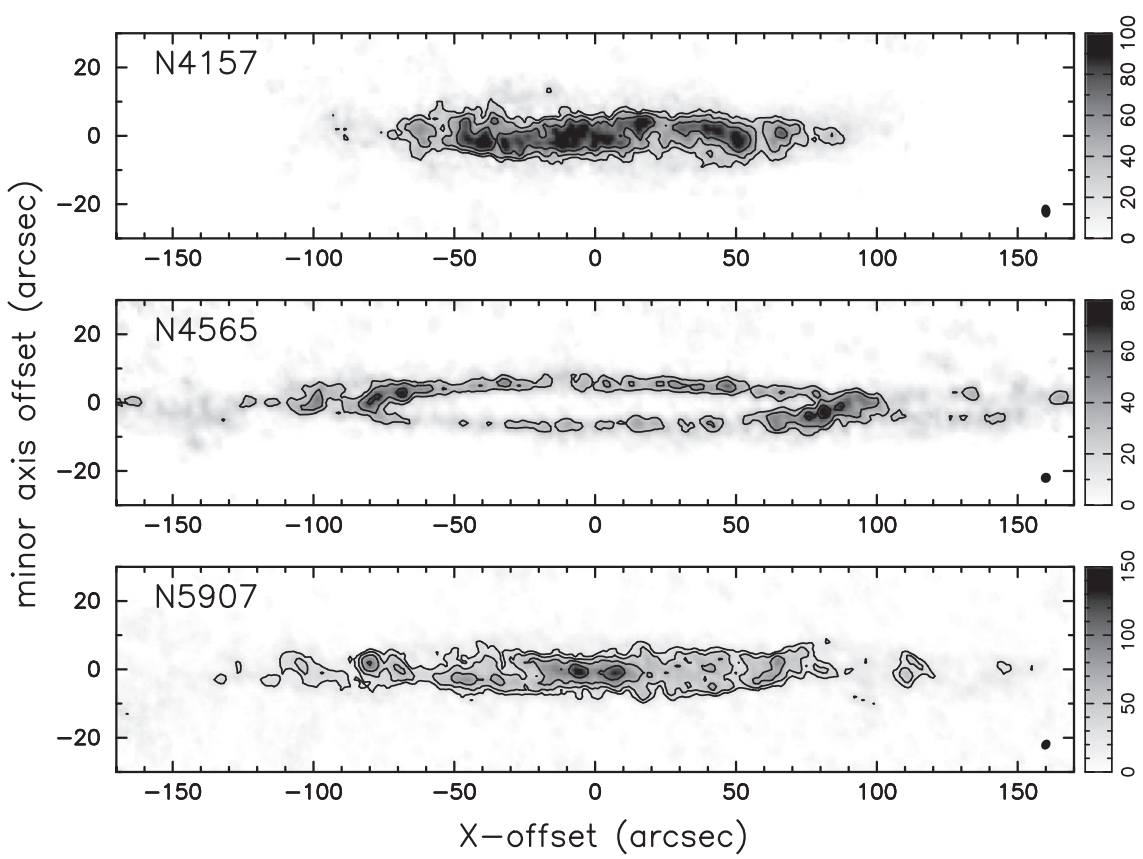

Figure 1. CO integrated intensity maps of NGC 4157 (top), NGC 4565 (middle), and NGC 5907 (bottom). Contour levels are $26 \times 1.4^{n} \mathrm{~K} \mathrm{~km} \mathrm{~s}{ }^{-1}$, with $n=0,1,2$, 3. The synthesized beam is shown in the lower right corner of each box.

\subsection{Hi Observations}

We have obtained $\mathrm{H}$ I data of the galaxies from the NRAO VLA archive. The array configurations are C and D for NGC 4157 and 5907 and B, C, and D for NGC 4565. A data reduction pipeline implemented in the CASA (Common Astronomy Software Applications) software package (McMullin et al. 2007) was built for reprocessing the whole data set in a uniform procedure, which we describe below.

For the data taken from each individual track, flagging of shadowed data was automatically performed first. Edge channels in each spectral window were also automatically flagged, if a test bandpass calibration showed their solution amplitudes falling below 0.75 . Any additional bad visibilities from either interference or instrumental problems were located and examined using tasks PLOTMS and VIEWER, and flagged using FLAGCMD with mode = "manualflag" or "quack." Then the flux calibrator visibility model was set using SETJY, and we computed the bandpass and flux-scaled gain tables from the primary and secondary calibrator data, using tasks BANDPASS, GAINCAL, and FLUXSCALE. Those solution tables were later applied to the data with the task APPLYCAL.

For the calibrated visibilities of each track, we usually performed a continuum subtraction in $u v$-space using the task UVCONTSUB. A first order polynomial fit was used to estimate the continuum emission from line-free channels. Then the data from different tracks were combined using the task CONCAT, and we imaged and cleaned the spectral data cubes using the CASA task CLEAN, with the ROBUST weighting algorithm (the ROBUST parameter was set to $R=0.5$ ). The clean depth went to a brightness level of 2.5 times the rms derived from the line free channels of a dirty cube.

Figure 2 shows the $\mathrm{H}$ I integrated intensity maps of NGC 4157,4565 , and 5907. The observing parameters including the angular and velocity resolutions are shown in Table 2 . The total flux in units of $\mathrm{Jy} \mathrm{km} \mathrm{s}^{-1}$ has been obtained from integration of the masked cube, where the mask was constructed from the $3 \sigma$ contour of a cube smoothed to $20^{\prime \prime}$. The average rms noise is measured in the line-free edge channels of the original cube. The strong warp of NGC 5907 noted in previous studies (e.g., Sancisi 1976) is evident. In addition, slight $\mathrm{H}_{\mathrm{I}}$ warps are shown in the other two galaxies (NGC 4157 and 4565). However, we do not see any evidence for warps in non-H I data for all three galaxies.

\subsection{IR Observations}

The IR data (IRAC $3.6 \mu \mathrm{m}$ and MIPS $24 \mu \mathrm{m}$ ) were obtained from the Spitzer archive: Program ID 69 (PI: G. Fazio) for both $3.6 \mu \mathrm{m}$ and $24 \mu \mathrm{m}$ images of NGC 4157 , Program ID 3 (PI: G. Fazio) for $3.6 \mu \mathrm{m}$ images of NGC 4565 and 5907, and Program ID 20268 (PI: R. de Jong) for $24 \mu \mathrm{m}$ images of NGC 4565 and 5907. We have downloaded the basic calibrated data (BCD) and used MOPEX (Mosaicking and Point Source Extraction) to remove background variations in the $\mathrm{BCD}$ images and mosaic the images. In the case of $24 \mu \mathrm{m}$, we have removed a residual flat field in the $\mathrm{BCD}$ data before background matching and mosaicking. In addition, we have downloaded $3.6 \mu \mathrm{m}$ images along with the supplied masks from the $S^{4} G$ archive (Sheth et al. 2010) and masked out the stars on the images for stellar modeling in Section 4.2.2.

The reduced $3.6 \mu \mathrm{m}$ and $24 \mu \mathrm{m}$ images of NGC 4157, 4565, and 5907 are shown in Figures 3 and 4, respectively. The ring seen in the CO map of NGC 4565 is clearly shown in the $24 \mu \mathrm{m}$ image. In the $3.6 \mu \mathrm{m}$ images, bright stars located nearby or projected against the galaxies are blanked and replaced by values from neighboring pixels using the Groningen Image Processing System (GIPSY; van der Hulst et al. 1992) tasks BLOT and PATCH before deriving their radial distributions. 


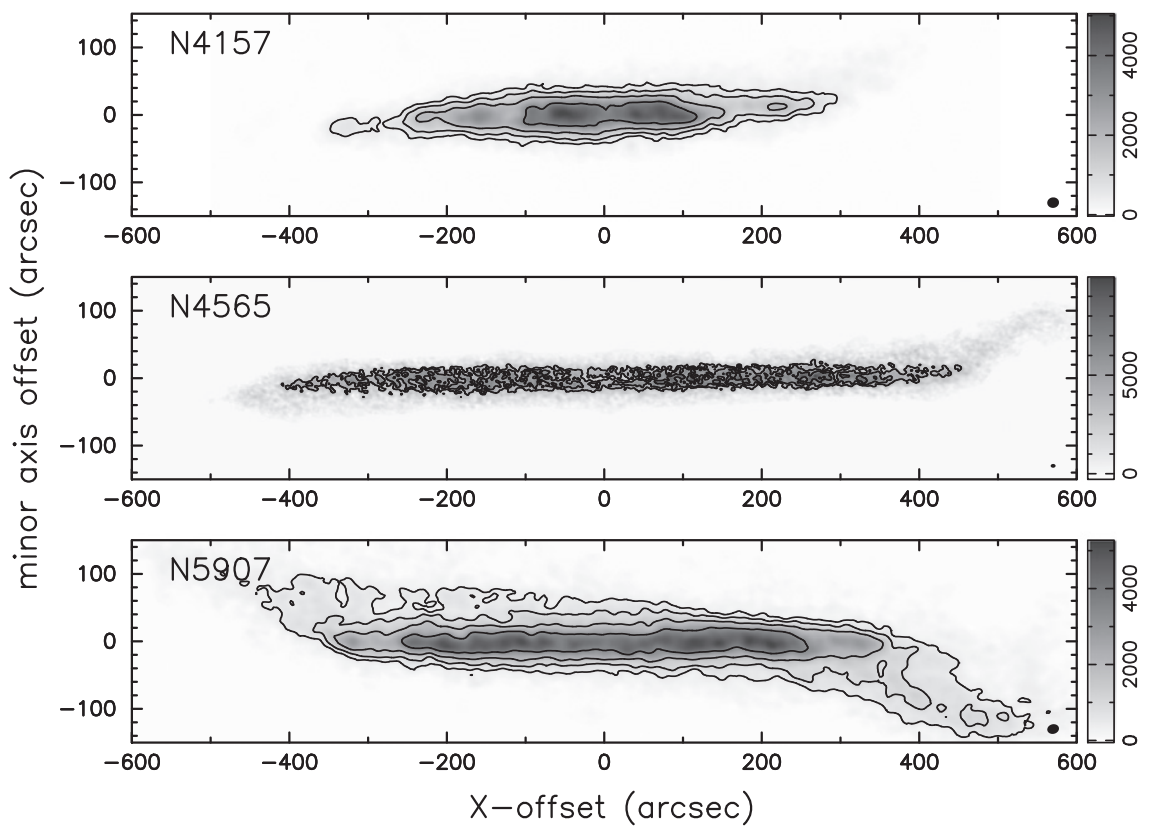

Figure 2. H I integrated intensity maps of NGC 4157 (top), NGC 4565 (middle), and NGC 5907 (bottom). Contour levels are $460 \times 1.9^{n} \mathrm{~K} \mathrm{~km} \mathrm{~s}{ }^{-1}$, with $n=0$, 1,2 , 3 for NGC 4157 and NGC 5907 and $3400 \times 1.4^{n} \mathrm{~K} \mathrm{~km} \mathrm{~s}^{-1}$, with $n=0,1,2,3$ for NGC 4565 . The synthesized beam is shown in the lower right corner of each box.

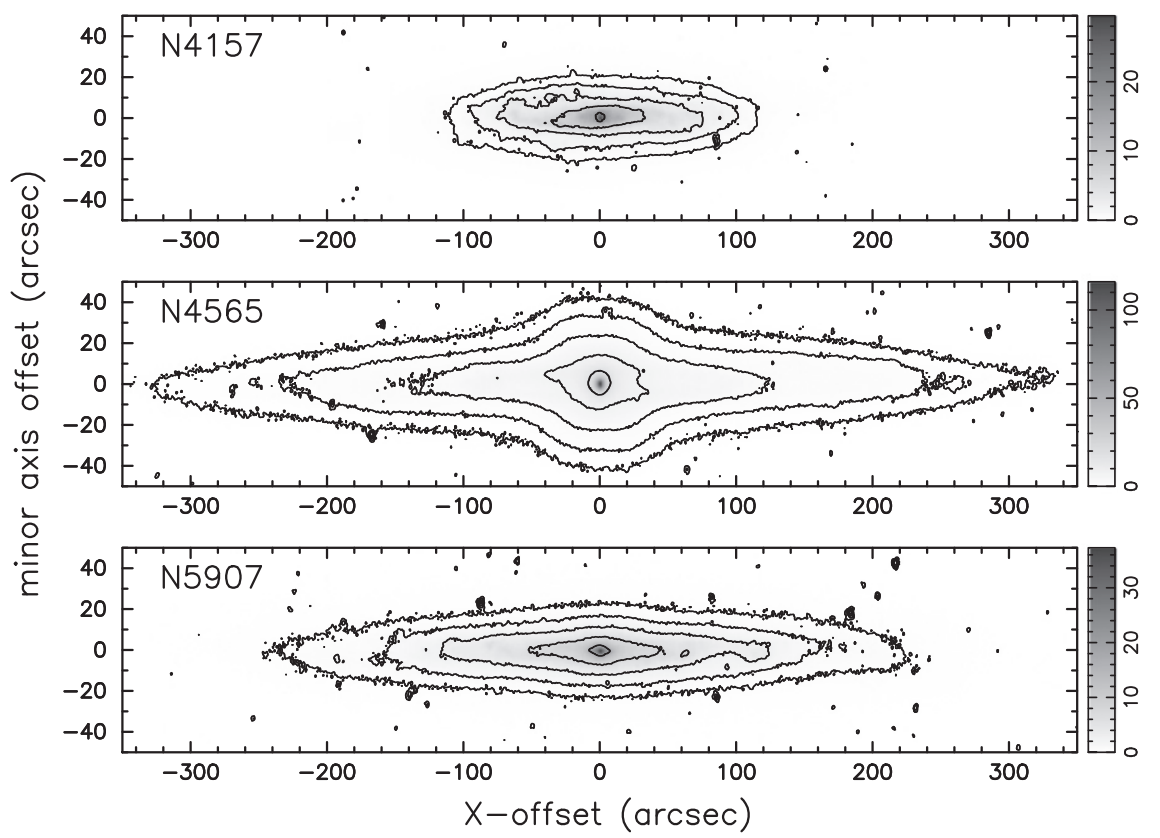

Figure 3. Spitzer $3.6 \mu \mathrm{m}$ images of NGC 4157 (top), NGC 4565 (middle), and NGC 5907 (bottom). Contour levels are $0.4 \times 2.6^{n} \mathrm{MJy} \mathrm{sr}^{-1}$, with $n=0,1,2,3,4$.

\section{RADIAL SURFACE DENSITY DISTRIBUTIONS}

\subsection{Molecular and Atomic Gas}

In order to derive the radial profile of gas surface density, we employ the position-velocity diagram (PVD) method from Paper I using vertically integrated position-velocity (p-v) diagrams (Figures 5, 6, and 7) and assuming circular rotation with a flat rotation curve (ignoring the rapidly rising velocity in the central region). The vertical integration for the $\mathrm{p}$ - $\mathrm{v}$ diagram extends between $10^{\prime \prime}$ above and below the midplane for $\mathrm{CO}$ and $\pm 50^{\prime \prime}$ for $\mathrm{H}_{\mathrm{I}}$. The central region in the $\mathrm{p}$-v diagrams (defined as a solid box in the figures), where contributions from many different radii overlap, is excluded when deriving the radial profile. At each pixel in the p-v diagram, the radius is obtained using the position $(x)$, the assumed circular speed $\left(V_{\mathrm{c}}\right)$, and the radial velocity $\left(V_{R}\right)$ :

$$
R=V_{\mathrm{c}}\left\langle\frac{x}{V_{R}-V_{\mathrm{sys}}}\right\rangle \quad \text { with }\left|\mathrm{V}_{R}-\mathrm{V}_{\mathrm{sys}}\right|<\mathrm{V}_{\mathrm{c}},
$$

where the angle brackets indicate the mean value of $x /\left(V_{R}-V_{\text {sys }}\right)$ within the pixel (see Paper I for more details) and the assumed circular speed is 220, 250, and $240 \mathrm{~km} \mathrm{~s}^{-1}$ (indicated as a dashed line in Figures 5-7) for 


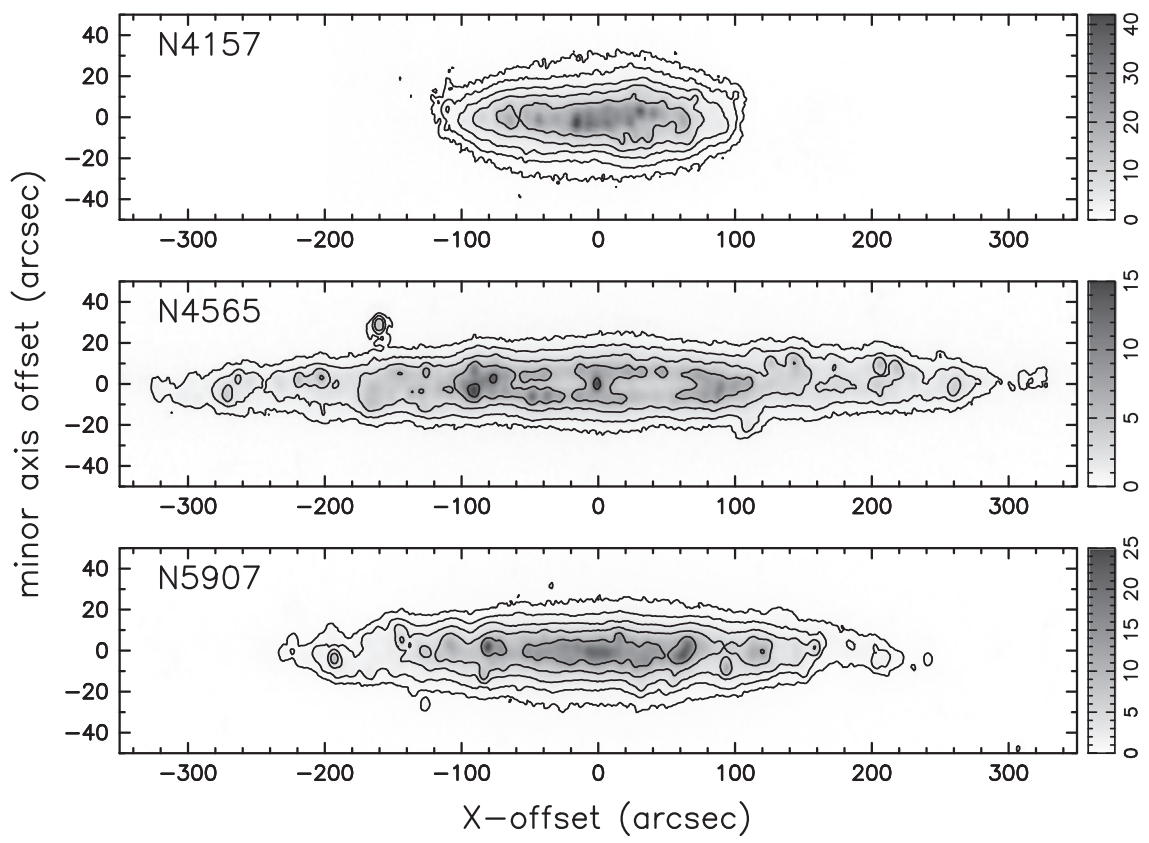

Figure 4. Spitzer $24 \mu \mathrm{m}$ images of NGC 4157 (top), NGC 4565 (middle), and NGC 5907 (bottom). Contour levels are $0.6 \times 2.1^{n} \mathrm{MJy} \mathrm{sr}^{-1}$, with $n=0,1,2,3,4$.
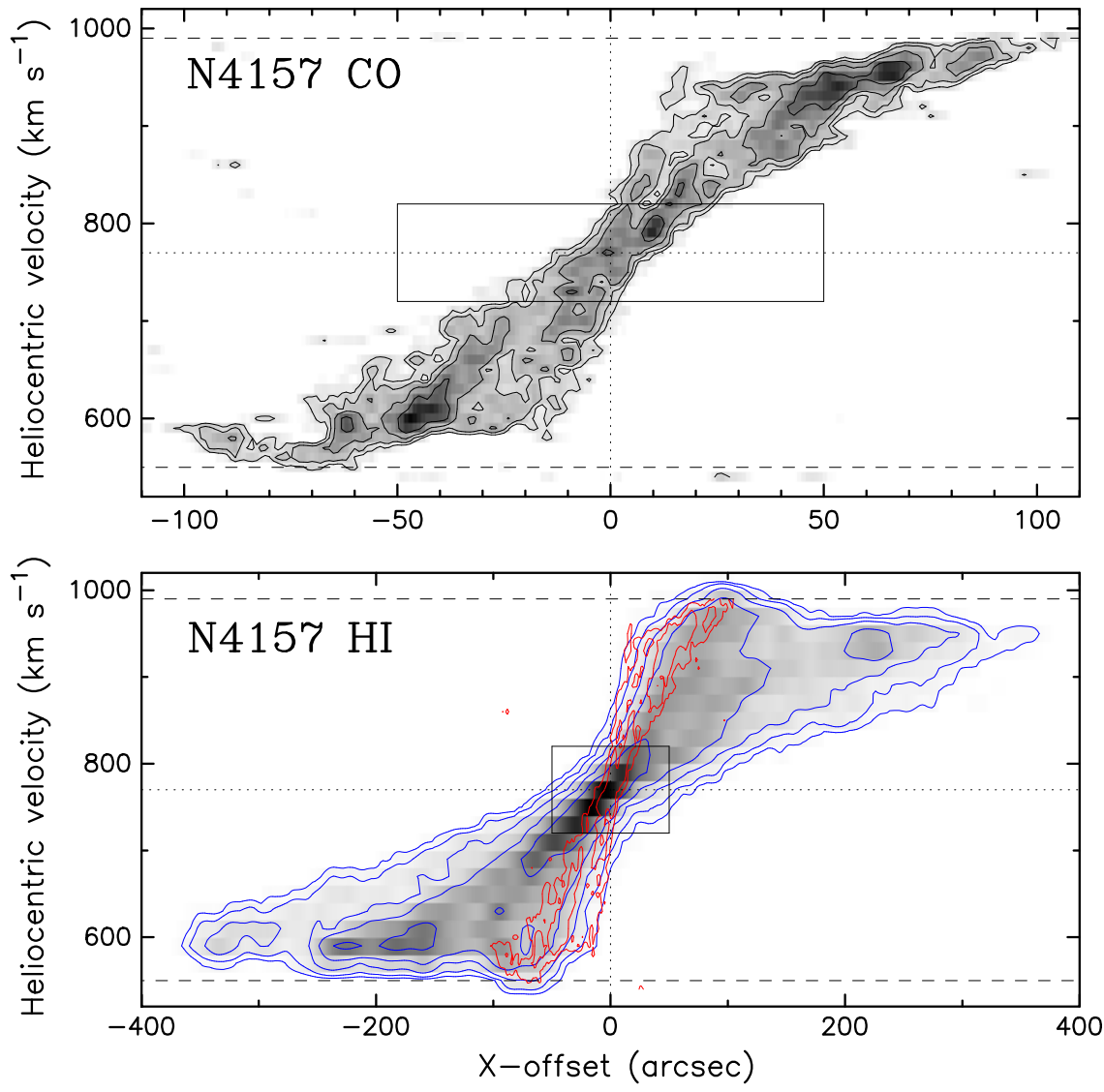

Figure 5. Vertically integrated position-velocity diagrams of NGC $4157 \mathrm{CO}$ (top) and $\mathrm{H}_{\mathrm{I}}$ (bottom). CO contours are $3.0 \times 1.7^{n} \mathrm{~K}$ arcsec, with $n=0,1,2,3$. $\mathrm{H}_{\mathrm{I}}$ contour levels are $93.0 \times 2.1^{n} \mathrm{~K}$ arcsec, with $n=0,1,2,3,4$. CO contours (red) are overlaid on H I contours (blue). The dashed lines show the assumed circular speed $\left(220 \mathrm{~km} \mathrm{~s}^{-1}\right)$ used to derive the radial profile using the PVD method. The box represents the excluded region in the PVD method.

(A color version of this figure is available in the online journal.)

NGC 4157, 4565, and 5907, respectively. The circular speed is obtained based on the maximum value of the rotation curve (Figure 8) that we derived using the envelope tracing method
(Sofue 1996), which is based on a p-v diagram along the midplane. In this method, the projected rotation curve $\left(V_{\text {proj }}\right)$ is derived from the terminal velocity $\left(V_{\mathrm{t}}\right)$ at each $x$ offset, 

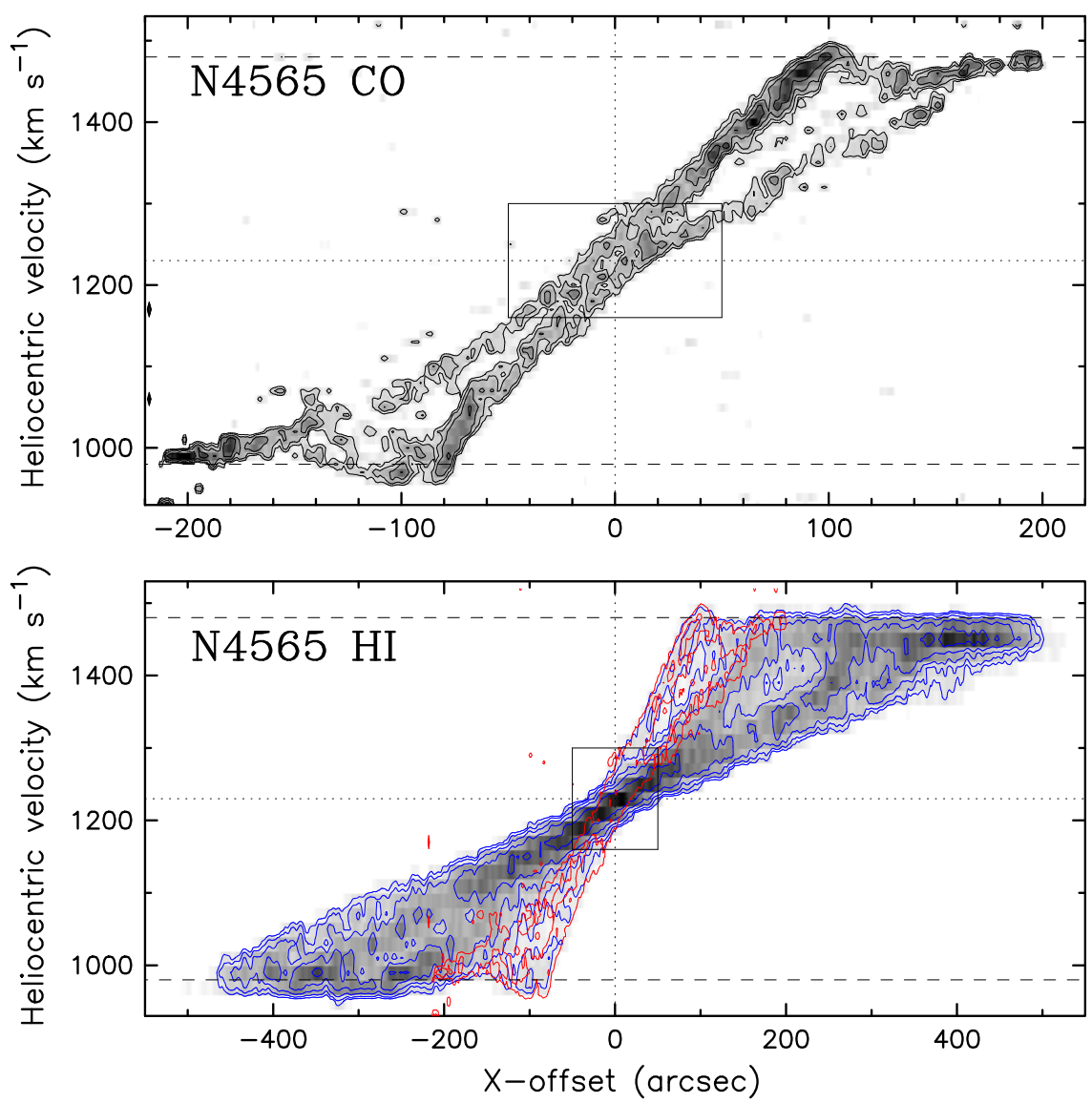

Figure 6. Vertically integrated position-velocity diagrams of NGC $4565 \mathrm{CO}$ (top) and $\mathrm{H}$ ( (bottom). CO contours are $2.2 \times 1.7^{n} \mathrm{~K}$ arcsec, with $n=0,1,2,3$. $\mathrm{H} \mathrm{I}$ contour levels are $450.0 \times 1.6^{n} \mathrm{~K}$ arcsec, with $n=0,1,2,3,4$. CO contours (red) are overlaid on $\mathrm{H}$ i contours (blue). The dashed lines show the assumed circular speed $\left(250 \mathrm{~km} \mathrm{~s}^{-1}\right)$ used to derive the radial profile using the PVD method. The box represents the excluded region in the PVD method.

(A color version of this figure is available in the online journal.)

considering the observational velocity resolution $\left(\sigma_{\mathrm{obs}}\right)$ and the gas velocity dispersion $\left(\sigma_{\mathrm{g}}\right)$ :

$$
V_{\text {proj }}=V_{\mathrm{t}}-\sqrt{\sigma_{\mathrm{obs}}^{2}+\sigma_{\mathrm{g}}^{2}},
$$

where the terminal velocity is selected as the highest velocity where the intensity exceeds the $3 \sigma$ level. The velocity resolutions for our observations are $10 \mathrm{~km} \mathrm{~s}^{-1}$ for $\mathrm{CO}$ data and $20 \mathrm{~km} \mathrm{~s}^{-1}$ for $\mathrm{H} \mathrm{I}$ data and the assumed velocity dispersion is $8 \mathrm{~km} \mathrm{~s}^{-1}$. Based on the correction term with $\sigma_{\mathrm{obs}}$ and $\sigma_{\mathrm{g}}$, we adopt an uncertainty for the rotation curve of $13 \mathrm{~km} \mathrm{~s}^{-1}$ for $\mathrm{CO}$ and $\sim 22 \mathrm{~km} \mathrm{~s}^{-1}$ for $\mathrm{H}_{\mathrm{I}}$. The derived surface brightnesses of $\mathrm{CO}$ and Hi by the PVD method (more details in Section 4.1 of Paper I) are converted to surface mass densities using the conversion factors shown in Equation (3) for CO (Strong \& Mattox 1996; Dame et al. 2001) and Equation (4) for $\mathrm{H}$ r:

$$
\begin{gathered}
N\left(\mathrm{H}_{2}\right)\left[\mathrm{cm}^{-2}\right]=2 \times 10^{20} I_{\mathrm{CO}}\left[\mathrm{K} \mathrm{km} \mathrm{s}^{-1}\right] . \\
N(\mathrm{HI})\left[\mathrm{cm}^{-2}\right]=1.82 \times 10^{18} I_{\mathrm{HI}}\left[\mathrm{K} \mathrm{km} \mathrm{s}^{-1}\right] .
\end{gathered}
$$

The derived radial profiles of $\Sigma_{\mathrm{HI}}, \Sigma_{\mathrm{H}_{2}}$, and the total gas $\left(\Sigma_{\mathrm{HI}}+\Sigma_{\mathrm{H}_{2}}\right)$ are shown in Figure 9 . The average value of both sides of the disk is used as the surface density profile in the figure and a factor of 1.36 for helium is included in the surface densities. For comparison between the molecular and atomic gas and to combine the two components for the total gas, the $\mathrm{CO}$ map has been convolved to the resolution of the H I map.
Vertical error bars show the standard deviation of the average value (of all data points) in a bin and horizontal error bars represent an uncertainty in the radius, showing obtainable maximum and minimum radii derived from varying $x$ and $V_{R}$ by the angular and the velocity resolutions. Some data points in the inner region of NGC 4565 are omitted since they are below the $3 \sigma$ detection threshold, which is obtained using the rms noise in the $\mathrm{p}-\mathrm{v}$ diagram. NGC 4565 shows a ring-like morphology as noticed in the CO map; NGC 4157 and 5907 exhibit small, centrally concentrated CO disks.

For comparison purposes, we also plot the radial profile of $\Sigma_{\mathrm{H}_{2}}$ obtained from the GIPSY task RADPROF (see Section 3.2 for details) as a magenta line in Figure 9. The RADPROF profile is in good agreement with the PVD profile of $\Sigma_{\mathrm{H}_{2}}$, although NGC 4565 exhibits differences between them in the central region, where $\mathrm{CO}$ emission is deficient and the uncertainties of the PVD profile are large. As discussed in Paper I, RADPROF is also subject to larger errors in the central region due to emission from outer radii seen in projection. In addition, it is unable to reproduce sharp gradients in the radial profile, such as those caused by gaps or rings.

\subsection{Stars and SFR}

We have used the RADPROF task to obtain radial distributions of the stellar and SFR surface densities since the IR data lack velocity information. The RADPROF task uses an 

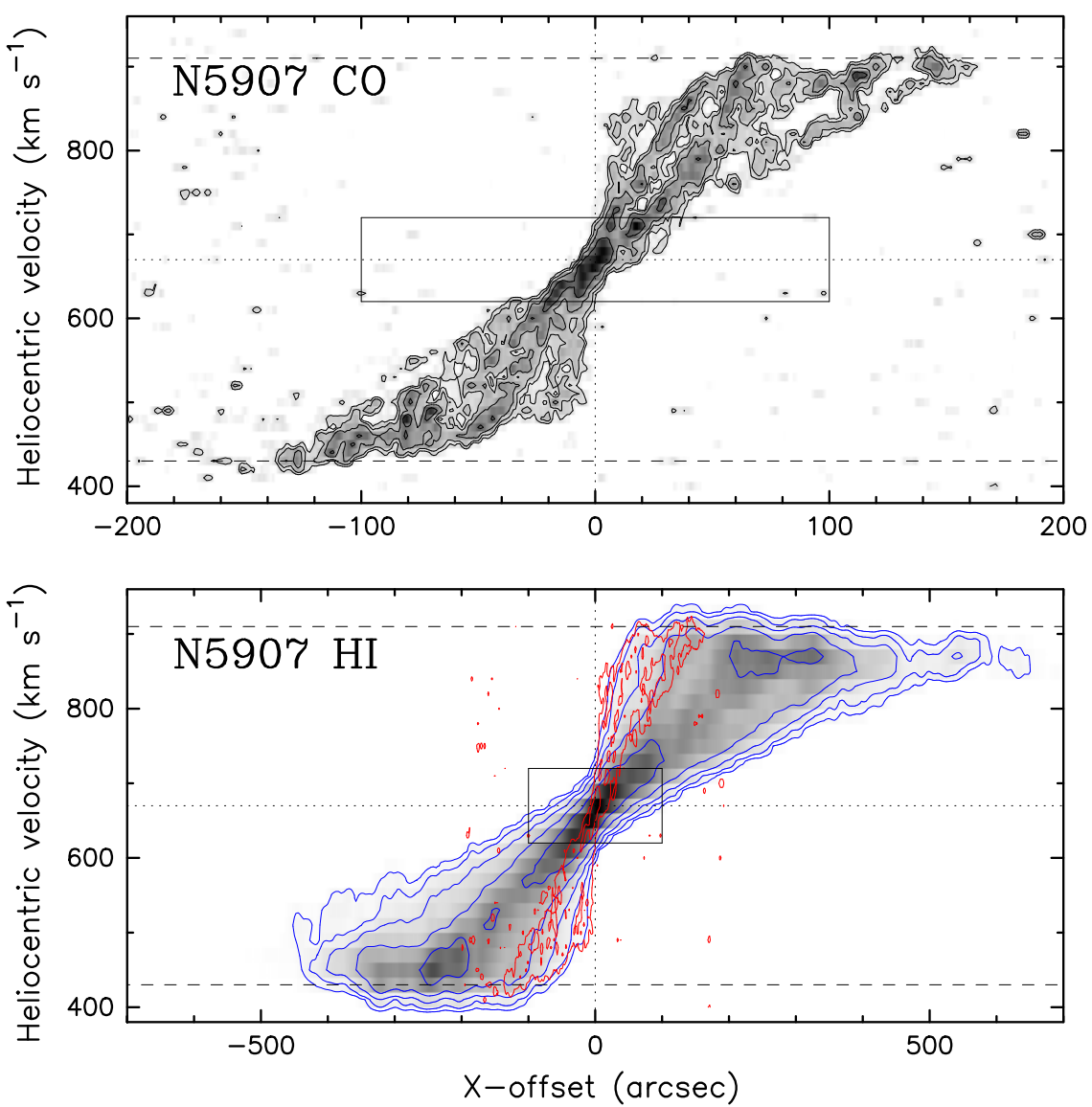

Figure 7. Vertically integrated position-velocity diagrams of NGC $5907 \mathrm{CO}$ (top) and $\mathrm{H}_{\mathrm{I}}$ (bottom). CO contours are $2.2 \times 1.7^{n} \mathrm{~K}$ arcsec, with $n=0,1,2,3$. $\mathrm{H} \mathrm{I}$ contour levels are $96.0 \times 2.1^{n} \mathrm{~K}$ arcsec, with $n=0,1,2,3,4$. CO contours (red) are overlaid on $\mathrm{H}_{\mathrm{I}}$ contours (blue). The dashed lines show the assumed circular speed $\left(240 \mathrm{~km} \mathrm{~s}^{-1}\right)$ used to derive the radial profile using the PVD method. The box represents the excluded region in the PVD method.

(A color version of this figure is available in the online journal.)

Abel inversion technique which assumes axisymmetry and requires a strip integral for each side of a galaxy, along with position angle, inclination (the values in Table 1 are used), beam size, and an initial function for the radial profile, which we assume to be exponentially decreasing for the $3.6 \mu \mathrm{m}$, and a Gaussian function for the $24 \mu \mathrm{m}$ data. Before employing the RADPROF task, all maps are smoothed to the lowest resolution among the data set. For better accuracy in comparison between SFR and gas, the $24 \mu \mathrm{m}$ maps were first convolved to a Gaussian beam of $7^{\prime \prime}$ using the IDL code and the kernel provided by Aniano et al. (2011) and then convolved to the $\mathrm{HI}$ beam except for NGC 4565 . Since the $\mathrm{H}_{\mathrm{I}}$ beam size of NGC 4565 is less than $7^{\prime \prime}$, other maps are convolved to the $24 \mu$ m Gaussian beam.

The derived $3.6 \mu \mathrm{m}$ radial profile from RADPROF is converted to stellar mass density using a conversion factor empirically derived by Leroy et al. (2008):

$$
\Sigma_{*}\left[M_{\odot} \mathrm{pc}^{-2}\right]=280(\cos i) I_{3.6}\left[\mathrm{MJy} \mathrm{sr}^{-1}\right],
$$

where the inclination $i$ is zero since the $3.6 \mu$ m intensity $\left(I_{3.6}\right)$ obtained from RADPROF is the face-on surface density. The assumed mass-to-light ratio ( $K$ band) for the conversion factor is 0.5 and the uncertainty quoted by Leroy et al. (2008) is about $0.1-0.2$ dex depending on galaxy color. The stellar radial distributions from RADPROF are shown as a blue line in Figure 10.
In order to estimate the effect of extinction on the stellar surface density, we have estimated the extinction at $3.6 \mu \mathrm{m}$ from the ratio of gas column density $N_{\mathrm{H}}$ to color excess $E(B-V)$ given by Bohlin et al. (1978) and the ratio of the extinction $A_{V}$ to $E(B-V) \sim 3.1$ (Rieke \& Lebofsky 1985):

$$
\frac{N_{\mathrm{H}}}{A_{V}} \approx 1.87 \times 10^{21} \text { atoms cm } \mathrm{cmag}^{-1} \text {. }
$$

Using the ratios $A_{3.6} / A_{K}=0.56$ (Indebetouw et al. 2005) and $A_{K} / A_{V}=0.112$ (Rieke \& Lebofsky 1985), the dust extinction at $3.6 \mu \mathrm{m}$ is:

$$
A_{3.6} \approx 3.35 \times 10^{-23} N_{\mathrm{H}} \text { mag. }
$$

Using the combined map of $\mathrm{CO}$ and $\mathrm{HI}$ as a proxy for gas column density, we have obtained an extinction corrected map and derived a radial profile from the map. The largest extinctions we measured through the midplane are $\sim 1 \mathrm{mag}$ for all the galaxies. By comparing the radial profile (Figure 10) with the extinction corrected profile, we noticed that the differences between two profiles are a factor of $\sim 2$ in the central regions for all the galaxies. In the outer regions, the difference is almost negligible. Note that the extinction correction is not applied to the stellar profile (Figure 10).

In addition to the RADPROF-derived stellar density profile, we also plot a fitted exponential disk model (red dashed line in the figure). The disk model we use is a self-gravitating and 

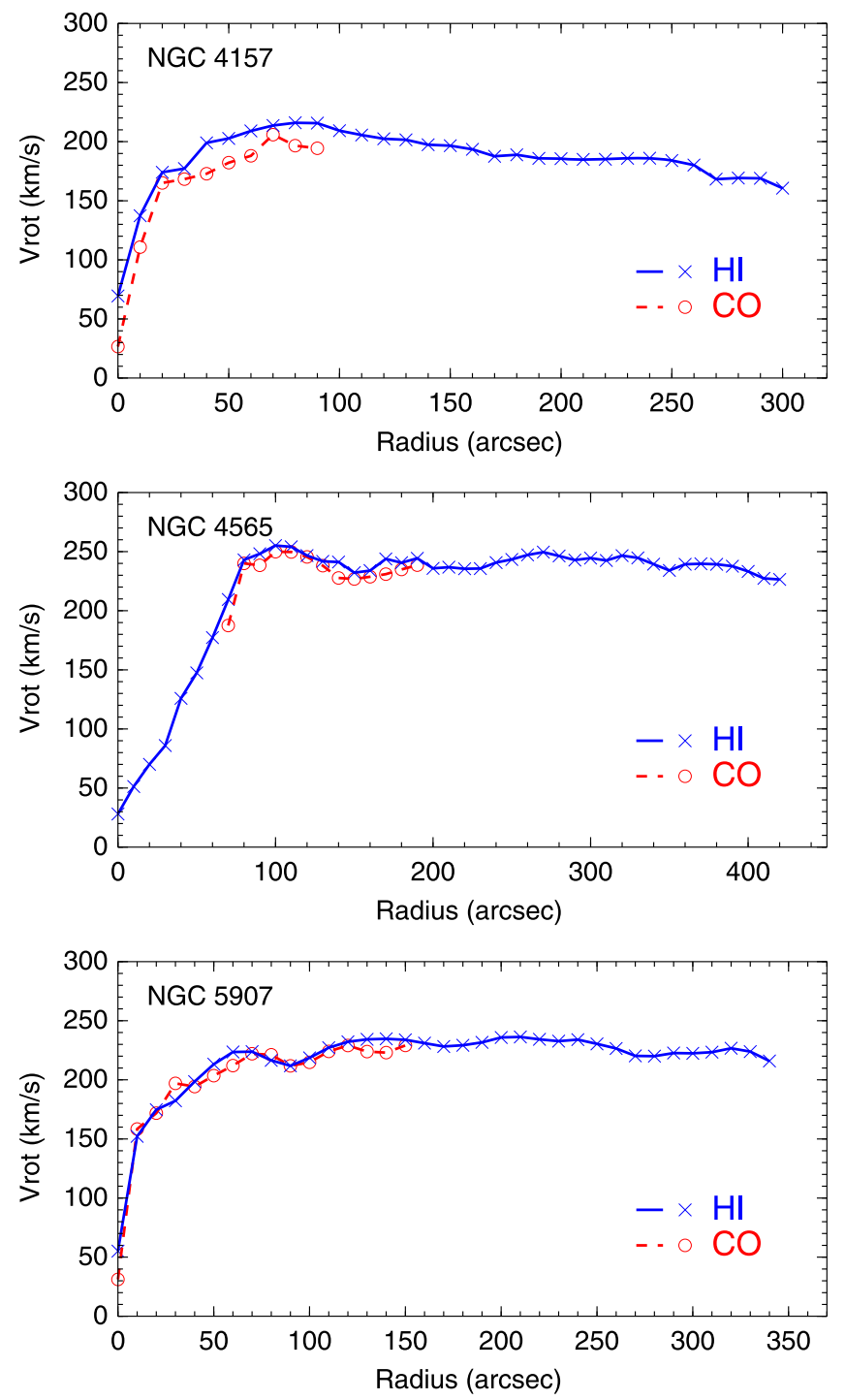

Figure 8. Rotation curves obtained from position-velocity diagrams along the midplane. The red circles show the CO rotation curve and blue crosses represent the $\mathrm{H}$ I rotation velocity.

(A color version of this figure is available in the online journal.) isothermal disk model provided by van der Kruit \& Searle (1981):

$$
L(R, z)=L_{0} e^{-R / l} \operatorname{sech}^{2}\left(\frac{z}{z_{*}}\right),
$$

where $L_{0}$ is the space luminosity density at the center and $l$ is the scale length. The vertically integrated luminosity density $L$ $(R)$ is converted to the mass density using Equation (5). For fitting, we use the projected intensity distribution for an edgeon galaxy, obtained by integrating the disk model:

$$
\mu(x, z)=\mu(0,0)\left(\frac{x}{l}\right) K_{1}\left(\frac{x}{l}\right) \operatorname{sech}^{2}\left(\frac{z}{z_{*}}\right)
$$

where $\mu(0,0)=2 l L_{0}$ and $K_{1}$ is the modified Bessel function of the second kind of order 1 . When fitting to the $3.6 \mu \mathrm{m}$ maps, the central regions (including the stellar bulge) are excluded: $|x|<10^{\prime \prime}$ for NGC 4157, $|x|<80^{\prime \prime}$ for NGC 4565, and $|x|<30^{\prime \prime}$ for NGC 5907. The scale lengths obtained by fitting Equation (9) are $\sim 32^{\prime \prime}$ or $\sim 2 \mathrm{kpc}(\mathrm{NGC} 4157), \sim 85^{\prime \prime}$ or $\sim 4$ $\mathrm{kpc}$ (NGC 4565), and $\sim 57^{\prime \prime}$ or $3 \mathrm{kpc}$ (NGC 5907). The scale heights $\left(z_{*}\right)$ are $\sim 12^{\prime \prime}$ or $\sim 780$ pc $($ NGC 4157$), \sim 14^{\prime \prime}$ or $\sim 640$ pc (NGC 4565$)$, and $\sim 13^{\prime \prime}$ or $\sim 670$ pc (NGC 5907). The vertical error bar in the lower left corner represents the uncertainty of the radial profile and is obtained from the largest difference between the RADPROF and the disk model profiles: 0.25 dex (NGC 4157), $0.10 \mathrm{dex}$ (NGC 4565), and $0.19 \mathrm{dex}$ (NGC 5907). Note that the excluded central regions are not considered when finding the largest difference for the uncertainty estimate.

The SFR surface density is determined from the Spitzer $24 \mu \mathrm{m}$ image by adopting the calibration given by Calzetti et al. (2007):

$$
\frac{\Sigma_{\mathrm{SFR}}}{M_{\odot} \mathrm{yr}^{-1} \mathrm{kpc}^{-2}}=1.56 \times 10^{-35}\left(\frac{S_{24 \mu \mathrm{m}}}{\mathrm{erg} \mathrm{s}^{-1} \mathrm{kpc}^{-2}}\right)^{0.8104}
$$
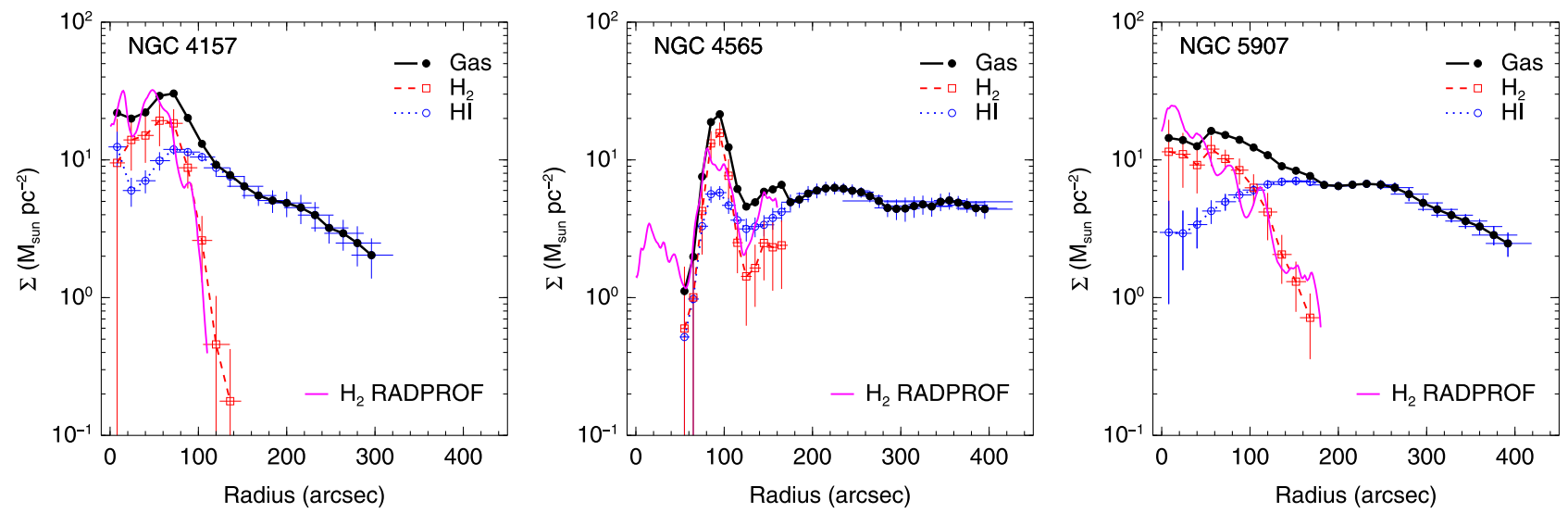

Figure 9. Radial distributions of $\mathrm{H}_{2}$ (red open squares), $\mathrm{H}$ ( (blue open circles), and total gas (solid circles) surface density for NGC 4157 (left), NGC 4565 (middle), and NGC 5907 (right). A factor of 1.36 is included in the surface densities for helium. The magenta line is the $\mathrm{H}_{2}$ radial profile obtained from RADPROF.

(A color version of this figure is available in the online journal.) 

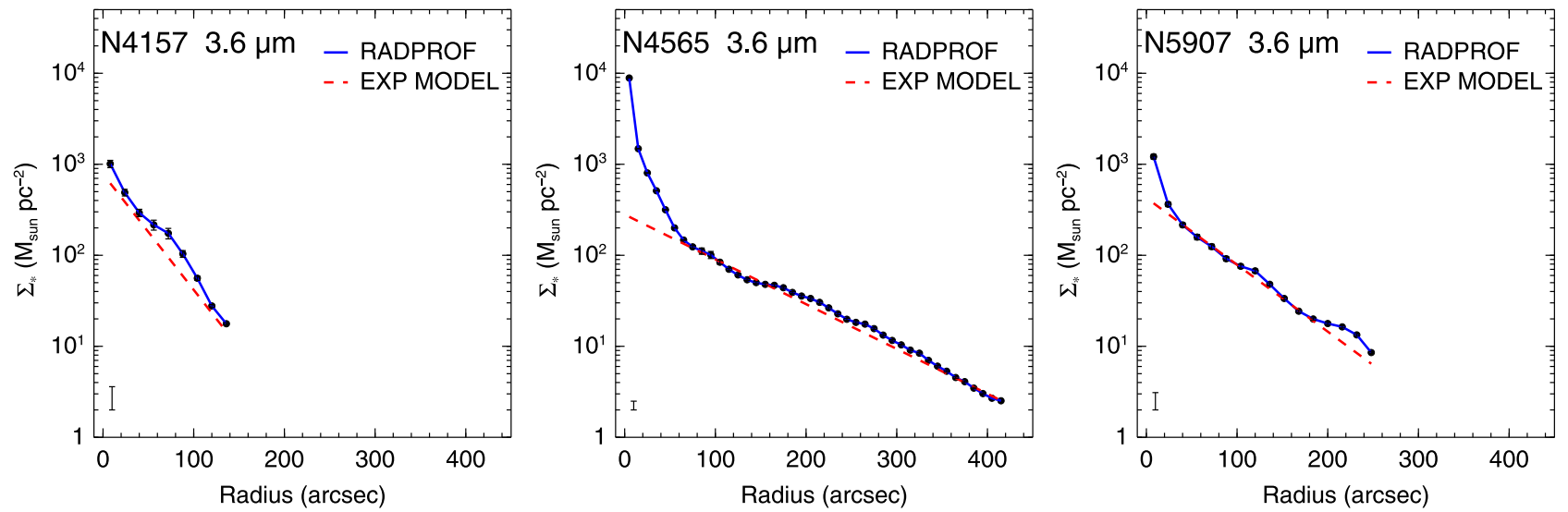

Figure 10. Stellar surface density as a function of radius for NGC 4157 (left), NGC 4565 (middle), and NGC 5907 (right). Spitzer $3.6 \mu$ m images are used to obtain the stellar radial profile. The blue solid line is the radial profile from the task RADPROF and the red dashed line shows the exponential disk model. The vertical error bar represents an uncertainty based on the largest difference between the RADPROF and the model profiles except the central regions excluded in the exponential fit. (A color version of this figure is available in the online journal.)
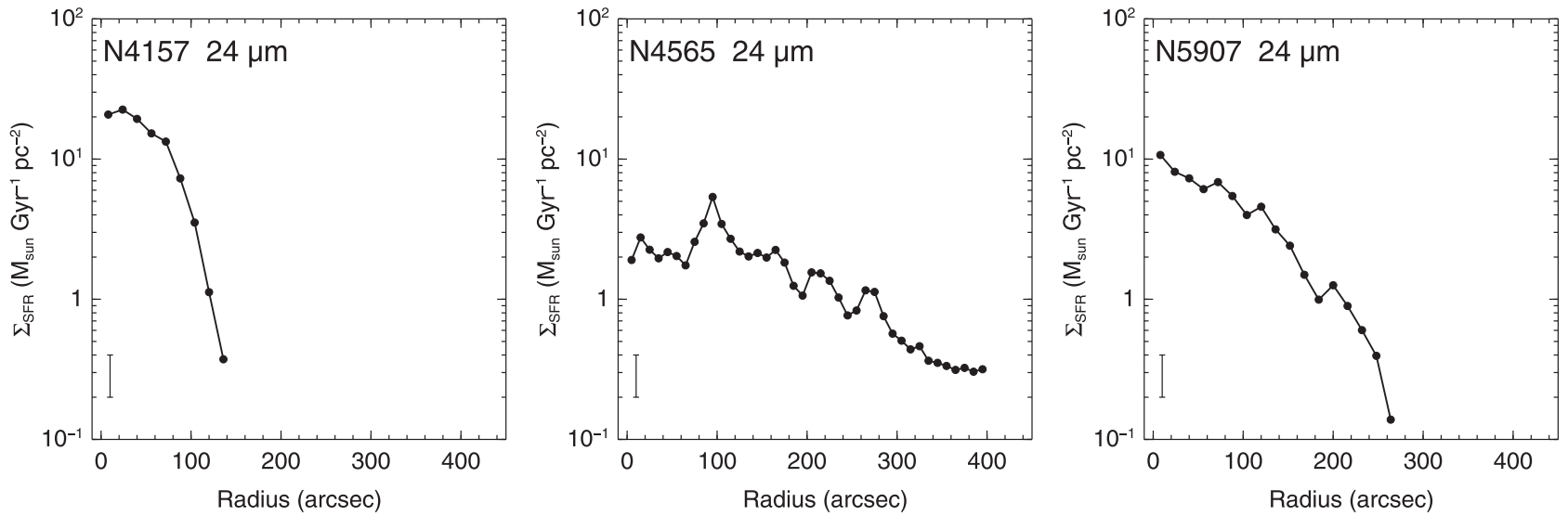

Figure 11. SFR surface density as a function of radius for NGC 4157 (left), NGC 4565 (middle), and NGC 5907 (right) obtained from Spitzer $24 \mu \mathrm{m}$ images using the task RADPROF.

where

$$
\frac{S_{24 \mu \mathrm{m}}}{\mathrm{erg} \mathrm{s}^{-1} \mathrm{kpc}^{-2}}=1.5 \times 10^{40}\left(\frac{I_{24}}{\mathrm{MJy} \mathrm{sr}^{-1}}\right) \text {, }
$$

and $I_{24}$ is the SFR $(24 \mu \mathrm{m})$ surface brightness derived from RADPROF.

Figure 11 shows the SFR radial profiles of the galaxies. The error bar, showing a factor of 2 uncertainty, is based on the largest difference between radial profiles from two different methods (RADPROF and ELLINT) for several face-on galaxies (observed by Spitzer at $24 \mu \mathrm{m}$ ) as described in Paper I. Before deriving the SFR radial profile of NGC 4565, the central compact source (possibly an AGN; Laine et al. 2010) in the $24 \mu \mathrm{m}$ map has been removed using the GIPSY tasks BLOT and PATCH. The SFR in these galaxies seems relatively low compared to the SFR in NGC 891 (Paper I). Dumke et al. (1997) also found that NGC 4565 and 5907 show lower star formation than NGC 891. The farinfrared luminosity at $40-400 \mu \mathrm{m}$ by IRAS (often used as a tracer for SFR) is $10.18,10.03,9.61,9.80$ in $\log _{10}\left(L / L_{\odot}\right)$ for NGC 891, 4157, 4565, and 5907, respectively (Sanders et al. 2003).

\section{RADIAL VARIATION IN VERTICAL STRUCTURE}

\subsection{Inclination and Gas Disk Thickness}

Unlike NGC 891, whose inclination is known to be $>89^{\circ}$ (Oosterloo et al. 2007), the other galaxies in our sample (NGC 4157, 4565, and 5907) appear less inclined. Since the inclination affects the apparent disk thickness, it is important to determine the inclination of the galaxies in order to obtain correct values for the scale height and the velocity dispersion.

We have derived inclinations and scale heights of these less edge-on galaxies via Olling's method (Olling 1996) which is described in detail in the Appendix. The derived inclinations with radius are shown in Figure 12 as blue solid and red open circles for $\mathrm{CO}$ and green solid and magenta open triangles for H I. Different colors indicate different halves of the galaxy. We show a weighted mean value of the inclination points as dotted $(\mathrm{CO})$ and dashed $\left(\mathrm{H}_{\mathrm{I}}\right)$ lines and adopt a representative value of $i=86.5^{\circ}$ for NGC 4565 and $i=86^{\circ}$ for NGC 5907 since their mean $\mathrm{CO}$ and $\mathrm{H}$ I values are very close. However, in the case of NGC 4157, the $\mathrm{CO}\left(84^{\circ}\right)$ and $\mathrm{HI}_{\mathrm{I}}\left(83^{\circ}\right)$ values show a difference, so we adopt the $\mathrm{CO}$ inclination value since the $\mathrm{H}$ I derived value may be affected by the outer warp. Even though there is a $1^{\circ}$ difference between $\mathrm{CO}$ and $\mathrm{H}_{\mathrm{I}}$ inclinations in NGC 4157, the values match well in the region where both tracers can be analyzed. We also note that the rotation curves of 

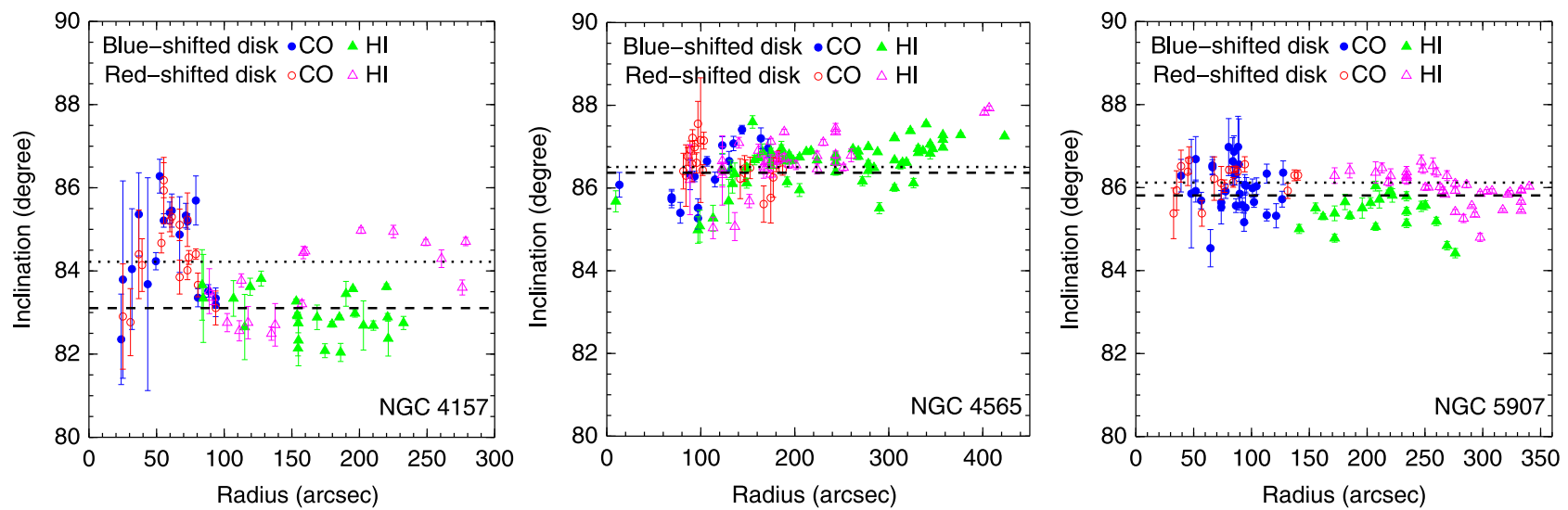

Figure 12. Inclinations as a function of radius for NGC 4157 (left), NGC 4565 (middle), and NGC 5907 (right). The blue solid and red open circles show the blueshifted disk and redshifted disk of $\mathrm{CO}$, respectively. The green solid and magenta open triangles represent the blue- and redshifted disks of $\mathrm{H}$, respectively. The horizontal dotted and dashed lines represent weighted mean values of the $\mathrm{CO}$ and $\mathrm{H}_{\mathrm{I}}$ inclinations, respectively.

(A color version of this figure is available in the online journal.)
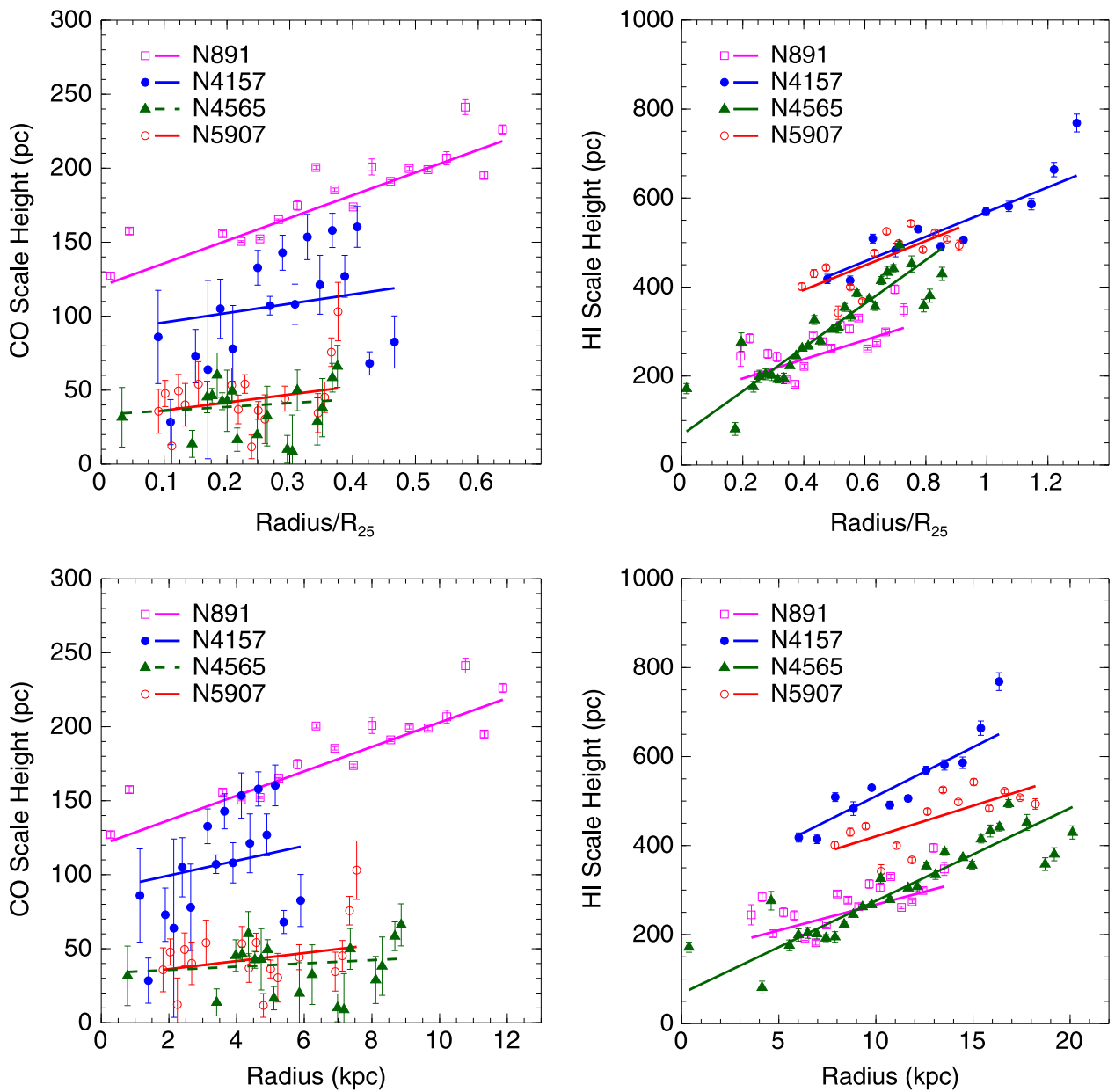

Figure 13. Top row: scale heights plotted against radius normalized by the optical radius $\left(R_{25}\right)$ for $\mathrm{CO}$ (left) and $\mathrm{H}$ ( (right). Bottom row: scale heights plotted against radius in units of kpc for $\mathrm{CO}$ and $\mathrm{H}$. The lines show linear approximations obtained by least-squares fitting.

(A color version of this figure is available in the online journal.)

$\mathrm{CO}$ and $\mathrm{H}_{\mathrm{I}}$ in this galaxy show a mismatch (especially in the region of $\left.30^{\prime \prime}-70^{\prime \prime}\right)$ unlike the other galaxies. Since the inclinations are determined using the distance $y_{\text {off }}$ (see the Appendix), which depends on the velocity field map, the mismatch between the rotation curves may contribute to the differences between the inclinations derived from $\mathrm{CO}$ and $\mathrm{H} \mathrm{I}$.
The measured scale heights (see the Appendix) considering the projection effects for the less edge-on galaxies are shown in Figure 13. We use the Gaussian width $(0.42 \times$ FWHM $)$ for the scale height. The scale heights for NGC 891 presented in Paper I have been included in this figure for comparison purposes. The lines show the linear least-squares fits to the data points 


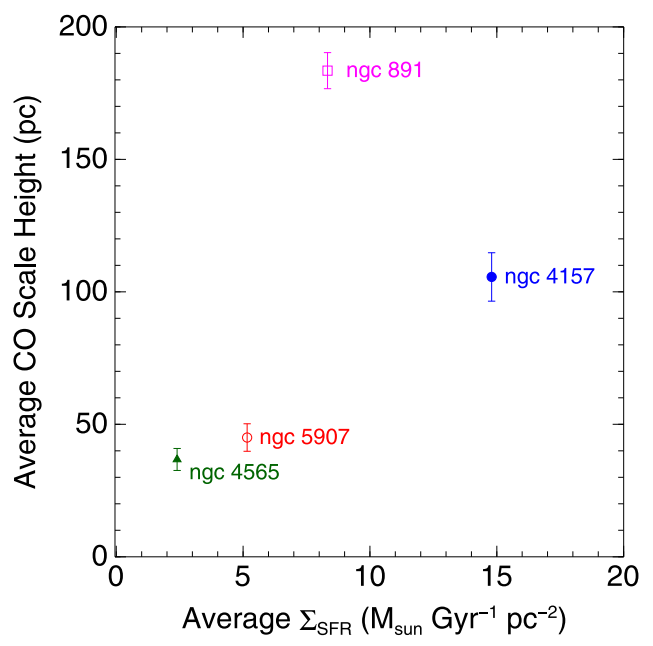

Figure 14. Average scale height of $\mathrm{CO}$ as a function of average $\Sigma_{\text {SFR }}$. The name of each galaxy is indicated next to its point. The vertical error bars are the standard deviation of the mean.

(A color version of this figure is available in the online journal.)

and we use the best fit line to derive the radial variation in the volume density and velocity dispersion in Sections 4.4 and 4.5. For the inner and outer regions where no data points are available, we assume a constant scale height within the innermost measured point when deriving the total midplane density and the total gas dispersion for the gravitational instability parameter $Q$ (Section 5.2) and the interstellar pressure (Section 6).

The thickness of the gas disk may be related to the star formation activity (Dumke et al. 1997 and references therein). From comparison between the CO disk thickness and the SFR surface density profile (Figure 11), we note that the galaxies with higher SFR (NGC 891 and NGC 4157) show a thicker $\mathrm{CO}$ disk. In order to investigate the relationship between the CO disk thickness and SFR, we plot the average CO scale height as a function of average $\Sigma_{\mathrm{SFR}}$ in Figure 14. The average $\Sigma_{\text {SFR }}$ values are obtained by integrating $\Sigma_{\text {SFR }}$ only over the CO disk range (i.e., the radial range over which estimates of the $\mathrm{CO}$ scale height are available) and normalizing by the disk area. This figure suggests a weak correlation between the galaxyaveraged $\mathrm{CO}$ disk thickness and the SFR. Within a galaxy, however, the two values are anti-correlated, with the CO disk being thicker in the outer galaxy where the SFR is lower.

\subsection{Stellar Disk Thickness}

Visual inspection of the $3.6 \mu \mathrm{m}$ images does not reveal clear indications of an increase in the scale height with radius. This may be due, however, to the rapidly decreasing surface brightness with radius and a viewing angle that is not exactly edge-on. We have therefore adopted an iterative approach to modeling the variation in stellar scale height.

\subsubsection{Initial Estimates}

Olling's method is not applicable for deriving the stellar scale height due to the lack of velocity information. In the case of NGC 891 (Paper I), we assumed a $\operatorname{sech}^{2}\left(z / h_{*}\right)$ function to derive the variation of the stellar scale height $\left(h_{*}\right)$ with radius. The vertical distributions at each radius was obtained by employing RADPROF to obtain radial distributions at many different vertical heights. For the less inclined galaxies studied here, however, projection effects must be taken into account. As an initial estimate for $h_{*}(R)$, we first employ the same RADPROF approach as in Paper I, then apply a multiplicative factor of $\sin i$ and make a least-squares linear fit to the resulting data points. The fitted line is then used to generate an initial disk model.

\subsubsection{Iterative Modeling}

In order to derive more accurate stellar scale heights, we generated a series of models in the GIPSY task GALMOD, fixing the inclination and radial surface density profile to derived values and allowing the scale height to vary for different models. We obtained minor axis profiles of the $3.6 \mu \mathrm{m}$ image and the models at various major axis $(x)$ offsets to derive the line-of-sight (LOS) projected scale height by fitting an exponential function to the vertical profiles. (An exponential function is what the $\operatorname{sech}^{2}$ function approaches at high z.) The central regions (including bulge) are excluded from the fitting. For galaxies except NGC 891, we use the $3.6 \mu \mathrm{m}$ images from the $\mathrm{S}^{4} \mathrm{G}$ archive (Sheth et al. 2010) in this procedure, and use the accompanying masks to exclude from the fitting any regions contaminated by point sources.

First, we generated an initial model as described in Section 4.2.1. Next, we compared the projected scale heights of the model cube and the $3.6 \mu \mathrm{m}$ map finding the modeled scale height to be an overestimate except for the almost edgeon galaxy NGC 891. We then input a new scale height into GALMOD estimated from the comparison between the initial model and the data. The new scale height has a much lower central value (about three times lower than the original value) and a similar slope. After comparing this second model to the data, we adjusted the parameters for the central value and the slope to obtain a subsequent model. This iteration was carried out until the modeled scale height reproduces the fit to the data. Following this scheme, we could find a reasonable model within seven trials.

The stellar scale height could be affected by uncertainties in the radial profile (which goes into the GALMOD modeling) so we checked the effects of extinction by starting with an extinction corrected radial profile. The new scale height from the extinction corrected density profile is increased by a factor of $\sim 1$ for all the galaxies; extinction does not appear to have a major effect on the scale height. But we are only considering the effect of extinction on the radial profiles, not the vertical profiles. The variation in the LOS-integrated scale heights have been compared with what has been observed in other edge-on galaxies given by de Grijs \& Peletier (1997); the gradients in our measurements are in the range of their sample.

From a comparison between the initial scale height based on the RADPROF method and the scale height of the best model we note the following. For the less edge-on galaxies, the initial central values from the data seem to be overestimated by a factor of $\sim 2-3$ compared to the values of the best models. The initial slopes of the scale heights are higher by a factor of $\sim 2$ for NGC 4157 and 5907 and 3 for NGC 4565 than those of the best model. On the other hand, the almost edge-on galaxy NGC 891 shows similar values between the best model and the data. We also verified that a constant scale height model produced LOS-integrated scale heights that were in significant disagreement with the data. We will use these scale heights obtained by the modeling when deriving the midplane volume 
Table 3

Parameters from Fitting the Radial Variation of the Scale Heights

\begin{tabular}{|c|c|c|c|c|c|c|c|}
\hline \multirow{2}{*}{ Galaxy } & \multirow{2}{*}{$\begin{array}{c}\text { Adopted } i \\
\left({ }^{\circ}\right)\end{array}$} & \multicolumn{3}{|c|}{ Central Value $(a)(\mathrm{pc})$} & \multicolumn{3}{|c|}{ Gradient $(b)(\mathrm{pc} / \mathrm{kpc})$} \\
\hline & & $\mathrm{CO}$ & $\mathrm{HI}_{\mathrm{I}}$ & Stars & $\mathrm{CO}$ & $\mathrm{H}_{\mathrm{I}}$ & Stars \\
\hline NGC 891 & 89 & $120 \pm 1$ & $151 \pm 5$ & 161 & $8.3 \pm 0.1$ & $11.6 \pm 0.5$ & 22.0 \\
\hline NGC 4565 & 86.5 & $33 \pm 8$ & $68 \pm 5$ & 165 & $1.1 \pm 1.4$ & $20.8 \pm 0.4$ & 20.0 \\
\hline NGC 5907 & 86 & $31 \pm 8$ & $284 \pm 8$ & 133 & $2.7 \pm 1.5$ & $13.7 \pm 0.6$ & 25.0 \\
\hline
\end{tabular}
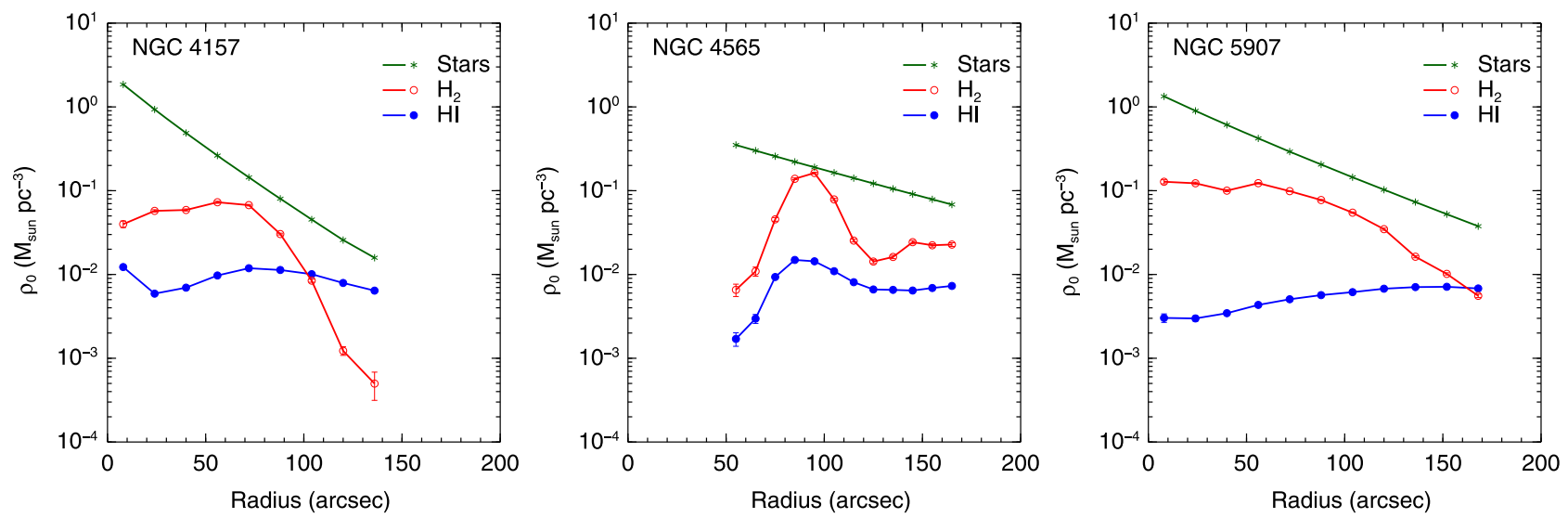

Figure 15. Midplane volume densities of $\mathrm{H}_{2}$ (red open circles), $\mathrm{H}_{\mathrm{I}}$ (blue solid circles), and stars (green asterisks) as a function of radius.

(A color version of this figure is available in the online journal.)

density and vertical velocity dispersion of stars in the following sections.

\subsection{Summary of Disk Thicknesses}

We characterize the radial variation in scale height using a linear function $h(R)$ expressed as:

$$
h=a+b \times R
$$

where the intercept $(a)$ and the slope $(b)$ with their uncertainties are summarized in Table 3. For stars, we show the parameters used to generate the best model. For ease of presentation, the slope is quoted in $\mathrm{pc} \mathrm{kpc}^{-1}$ rather than in dimensionless form. Unlike the scale heights of $\mathrm{HI}$, the uncertainties in the slopes of the $\mathrm{CO}$ scale heights are very large in the three galaxies NGC 4157, 4565, and 5907 and in the case of NGC 4565 the measured gradient is not significant. However, most galaxies show increases with radius in the scale heights of $\mathrm{CO}, \mathrm{H}$, and stars.

\subsection{Midplane Volume Densities}

Using the derived surface mass densities and the scale heights from the previous sections, we have obtained the midplane volume density $\left(\rho_{0}\right)$ profiles with radius:

$$
\begin{aligned}
\rho_{0 \mathrm{H}_{2}} & =\frac{\Sigma_{\mathrm{H}_{2}}}{h_{\mathrm{H}_{2}} \sqrt{2 \pi}}, \quad \rho_{0 \mathrm{HI}}=\frac{\Sigma_{\mathrm{HI}}}{h_{\mathrm{HI}} \sqrt{2 \pi}}, \\
\rho_{0 *} & =\frac{\Sigma_{*}}{2 h_{*}} .
\end{aligned}
$$

Here we assume a Gaussian distribution for the gas $\left(\mathrm{H}_{2}\right.$ and $\left.\mathrm{H}_{\mathrm{I}}\right)$ and $\operatorname{sech}^{2}\left(z / h_{*}\right)$ function for stars. The estimated volume densities are shown in Figure 15. The uncertainties shown in the figure include errors in the radial profile but not in the scale height or the CO-to- $\mathrm{H}_{2}$ conversion factor. A factor of 2 variation in the $X_{\mathrm{CO}}$ factor would lead to a similar variation in the volume density. This midplane density profile allows us to explore the role that the turbulent interstellar pressure $\left(\rho_{0} \sigma_{\mathrm{g}}^{2}\right)$ plays in controlling the molecular to atomic (volume) gas density ratio, in comparison with the relationship between the hydrostatic midplane pressure and the $\mathrm{H}_{2} / \mathrm{H}_{\mathrm{I}}$ ratio based on surface mass density. In addition, the density profile will allow us to investigate the SFL in terms of volume density instead of surface density, although we defer this analysis to the next paper in this series.

\subsection{Vertical Velocity Dispersion}

Since it is not easy to measure a gaseous vertical velocity dispersion directly, some earlier studies had suggested fairly constant values (Combes \& Becquaert 1997; Petric \& Rupen 2007). As an alternative, we have inferred the velocity dispersions as a function of radius using a numerical solution to the Poisson equation for a multi-component disk (Narayan \& Jog 2002):

$$
\sigma_{i}^{2}=\frac{4 \pi G \rho_{0, \text { tot }} \rho_{0 i}}{-\left(d^{2} \rho_{i} / d z^{2}\right)_{z=0}},
$$

where the subscript $i$ can refer to either g (gas) or * (stars):

$$
\begin{aligned}
& d^{2} \rho_{\mathrm{g}} / d z^{2}=-\rho_{0 \mathrm{~g}} / h_{\mathrm{g}}^{2}, \quad d^{2} \rho_{*} / d z^{2}=-2 \rho_{0 *} / h_{*}^{2} \\
& \text { at } z=0 \text {, } \\
& \Rightarrow \sigma_{\mathrm{g}}=\sqrt{4 \pi G h_{\mathrm{g}}^{2} \rho_{0, \mathrm{tot}}}, \quad \sigma_{*}=\sqrt{2 \pi G h_{*}^{2} \rho_{0, \text { tot }}} .
\end{aligned}
$$

Here the total midplane density is 

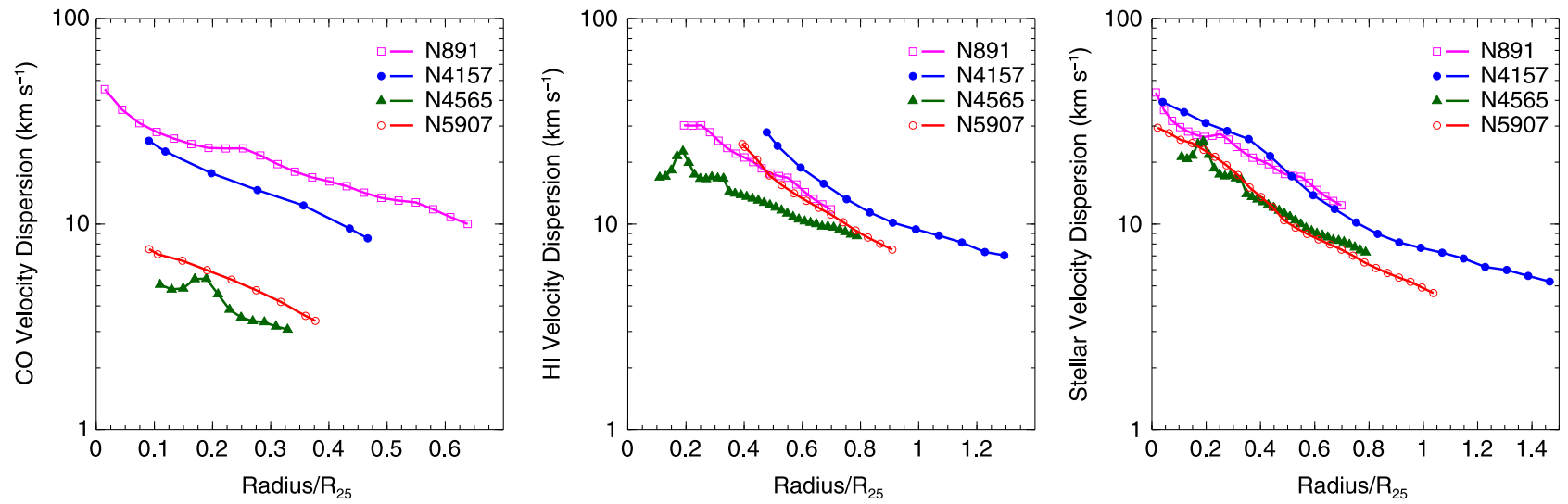

Figure 16. Vertical velocity dispersions against radius normalized by the optical radius $\left(R_{25}\right)$ for $\mathrm{CO}$ (left), $\mathrm{H}_{\mathrm{I}}(\mathrm{middle})$, and stars (right).

(A color version of this figure is available in the online journal.)
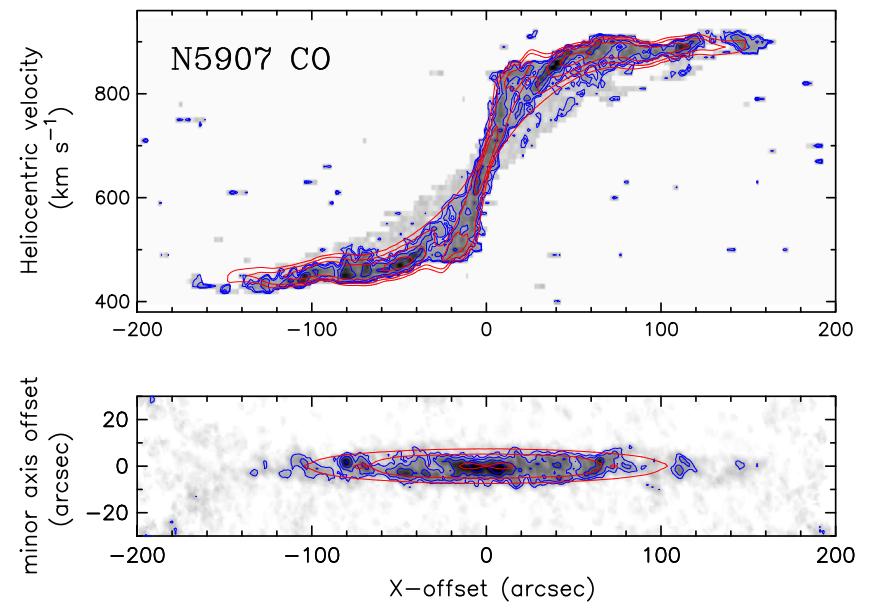

Figure 17. CO position-velocity diagram along the major axis (top) and integrated intensity map of NGC 5907 (bottom). TiRiFiC model contours (red) are overlaid on the map contours (blue). Both contour levels are $0.2 \times 1.9^{n}$ $\mathrm{K}$ arcsec, with $n=0,1,2,3$ (top) and $26 \times 1.4^{n} \mathrm{~K} \mathrm{~km} \mathrm{~s}^{-1}$, with $n=0,1,2,3$ (bottom).

(A color version of this figure is available in the online journal.)

$$
\rho_{0, \text { tot }}=\rho_{0 \mathrm{H}_{2}}+\rho_{0 \mathrm{HI}}+\rho_{0 *} \cdot
$$

We ignore the dark matter halo under the assumption that the dark matter density in the disk is relatively small compared to that of the stars. The boundary conditions we have used are $\rho_{i}=\rho_{0 i}$ and $d \rho_{i} / d z=0$ at the midplane $(z=0)$.

The velocity dispersions of $\mathrm{CO}, \mathrm{H}_{\mathrm{I}}$, and stars for the galaxies including NGC 891 are shown in Figure 16. The two galaxies NGC 4565 and 5907 show very low values of the CO velocity dispersion compared to the other galaxies. The two main reasons for the low velocity dispersions are because of low values in (1) the total midplane density and (2) the scale height of $\mathrm{CO}$ (see Equation 16). On the other hand, the velocity dispersions of $\mathrm{H}$ I and stars show similar values (within a factor of $\sim 2$ ) and trends for all galaxies. Note that when we estimate the midplane total density to derive the $\mathrm{H}_{\mathrm{I}}$ and stellar velocity dispersions, the molecular densities in the regions outside the $\mathrm{CO}$ field of view are assumed to be zero-a reasonable approximation because these regions are strongly $\mathrm{H}_{\mathrm{I}}$ dominated (with the possible exception of NGC 4565). In general, our results show that the velocity dispersions of $\mathrm{CO}, \mathrm{HI}$, and stars decrease as a function of radius.

\subsection{Tilted Ring Fitting Code (TiRiFiC) Modeling}

In order to verify the derived scale heights and velocity dispersions of the gas, we have built $\mathrm{CO}$ and $\mathrm{H}$ i models of NGC 5907 (as a representative example) using the TiRiFiC (Józsa et al. 2007) and compared the models with the data. The main input parameters we used for modeling in TiRiFiC are the derived rotation curve, density profile, scale height, velocity dispersion, and inclination. From the comparisons of the midplane and $z$-integrated $\mathrm{p}-\mathrm{v}$ diagrams and the integrated intensity maps (Figure 17), we confirmed that the gas models match the data reasonably well although the model does not include the $\mathrm{H}_{\mathrm{I}}$ warp. It suggests that the derived $\mathrm{CO}$ and $\mathrm{HI}_{\mathrm{I}}$ scale heights and velocity dispersions as well as the rotation curves are consistent with the data.

\section{STAR FORMATION}

In this section, we examine the $\mathrm{K}-\mathrm{S}$ law (SFL in terms of surface density) in our edge-on galaxy sample and compare the obtained $\mathrm{K}-\mathrm{S}$ law indices with values from face-on systems found in other studies. We also test the role of the gravitational instability parameter $Q$ in determining the region where stars form. We are unable to derive volume density estimates for the SFR from the present data so we defer discussion of the volumetric SFL to a future study.

\section{1. $K-S$ Law}

In Figure 18, we plot $\Sigma_{\mathrm{SFR}}$ versus $\Sigma_{\mathrm{H}_{2}}$ (left) and $\Sigma_{\text {gas }}$ (right) for our galaxy sample including NGC 891 in order to examine the strength of the relationships and compare their slopes. The uncertainties on the SFR and the gas surface densities are shown in their radial profiles in Section 3. In addition, about a factor of 2 uncertainty in the $\mathrm{CO}-$ to- $\mathrm{H}_{2}$ conversion factor (e.g., Bolatto et al. 2013) and the $24 \mu \mathrm{m}$ SFR calibration (e.g., Murphy et al. 2012) are suggested by the literature. We use the ordinary least-squares (OLS) bisector (Isobe et al. 1990) to fit the relationships between log quantities. The obtained powerlaw index and rms scatter (dex) around the fit for all galaxies are presented in Table 4. Note that the index of NGC 891 is a bit different from Paper I since we use the OLS bisector instead of the least-squares fitting used in Paper I. In addition, the index of NGC 891 for the total gas is much higher (by a factor of 2) than the index in Paper I because we use gas data points up to $R=400^{\prime \prime}$, instead of $300^{\prime \prime}$ in Paper I. Our results suggest 

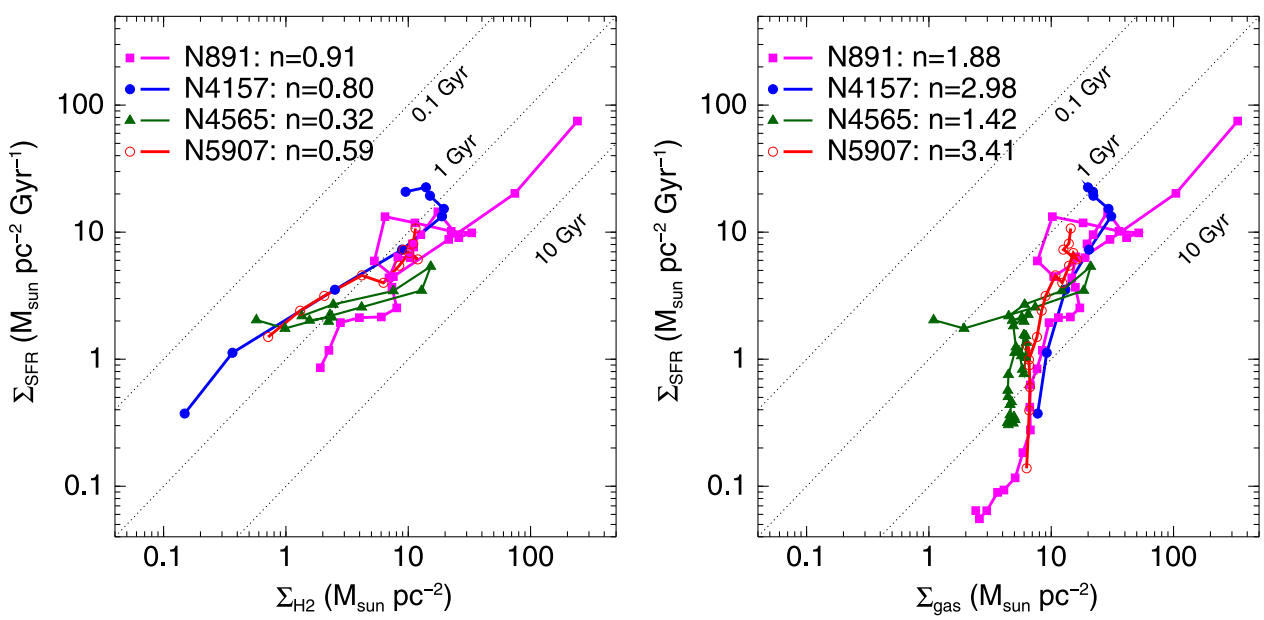

Figure 18. Relationship between $\Sigma_{\mathrm{SFR}}$ and $\Sigma_{\mathrm{H}_{2}}$ (left) and between $\Sigma_{\mathrm{SFR}}$ and $\Sigma_{\text {gas }}$ (right) for NGC 891, 4157, 4565, and 5907. The power-law index (K-S law index) is indicated in the upper-left corner. The dashed line shows constant SFE, with the gas depletion time (1/SFE) labeled.

(A color version of this figure is available in the online journal.)

Table 4

Power-law Indices for the Star Formation Law

\begin{tabular}{lccccc}
\hline \hline \multirow{2}{*}{ Galaxy } & \multicolumn{2}{c}{ Molecular Gas } & & \multicolumn{2}{c}{ Total Gas } \\
\cline { 2 - 3 } \cline { 5 - 6 } & Index & rms Scatter & & Index & rms Scatter \\
\hline NGC 891 & 0.91 & 0.19 & & 1.88 & 0.42 \\
NGC 4157 & 0.80 & 0.15 & & 2.98 & 0.25 \\
NGC 4565 & 0.32 & 0.06 & & 1.42 & 0.36 \\
NGC 5907 & 0.59 & 0.08 & & 3.41 & 0.23 \\
\hline
\end{tabular}

that the correlation between $\Sigma_{\mathrm{SFR}}$ and $\Sigma_{\mathrm{H}_{2}}$ is better than the correlation between $\Sigma_{\mathrm{SFR}}$ and $\Sigma_{\text {gas }}$ when considering the rms scatter around the relationship. In addition, most individual relations between $\Sigma_{\mathrm{SFR}}$ and $\Sigma_{\text {gas }}$ appear to show distinct slopes at the low and high ends; this is most noticeable in NGC 4565.

The most frequently cited relation given by Kennicutt (1998) suggests an index of 1.4: $\Sigma_{\text {SFR }} \propto \Sigma_{\text {gas }}^{1.4}$. Wong \& Blitz (2002) obtained weighted average indices of 0.78 (molecular gas) and 1.12 (total gas) from seven spiral galaxies. Leroy et al. (2005) found an index of 1.3 for the relationship between SFR (traced by the radio continuum) and $\mathrm{H}_{2}$ in dwarf galaxies. Bigiel et al. (2008) derived average values of 0.96 for $\mathrm{H}_{2}$ and 1.85 for $\mathrm{H} \mathrm{I}+\mathrm{H}_{2}$ from seven spiral galaxies. Bigiel et al. (2008) also obtained a smaller scatter for the molecular gas relation $(0.2 \mathrm{dex})$ than for the total gas relation $(0.3 \mathrm{dex})$. The overall tendency toward a steeper index in the correlation between total gas and SFR compared to the "molecular SFL" $\left(\Sigma_{\mathrm{SFR}} \propto \Sigma_{\mathrm{H}_{2}}{ }^{\mathrm{n}}\right)$ is also shown in our results since the relatively constant values in the atomic gas density profile steepen the total gas SFL $\left(\Sigma_{\mathrm{SFR}} \propto \Sigma_{\mathrm{gas}}{ }^{\mathrm{n}}\right)$. However, the derived "molecular" SFL indices are generally smaller $(0.32-0.91)$ than found in previous studies, especially in NGC 4565 and 5907. Among the reasons for the shallow slopes in our results might be optical depth effects in the $24 \mu \mathrm{m}$ emission which decreases the $24 \mu \mathrm{m}$ brightness in bright regions or contribution from diffuse starlight heating which increases the $24 \mu \mathrm{m}$ brightness in faint regions. As mentioned in Section 3.2, we have explored the opacity issue on $3.6 \mu \mathrm{m}$ and obtained the uncertainty of a factor of 2 by the extinction effects. Since the $24 \mu \mathrm{m}$ opacity should be less than the $3.6 \mu \mathrm{m}$ opacity, we expect less than a factor of 2 increase in the central region at the $24 \mu$ m radial profile. When we applied the uncertainty by the extinction at $3.6 \mu \mathrm{m}$ to the SFR radial profile, the indices for $\mathrm{H}_{2}$ are increased by a factor of $\sim 1.2$ (NGC 4157), 1.4 (NGC 4565), and 1.2 (NGC 5907). Nevertheless, the index of NGC 4565 is still smaller, but it is not unexpected since the CO emission of NGC 4565 is deficient in the central regions as shown in the CO map (ring-like morphology) and the gradient of the $24 \mu \mathrm{m}$ profile is flatter than the others. In addition, NGC 4565 has an AGN, which can heat the dust to produce $24 \mu \mathrm{m}$ even without much star formation. Rahman et al. $(2011,2012)$ have suggested that one possible reason for the flatter slopes in some galaxies among their sample might be a greater contribution from diffuse emission to the $24 \mu \mathrm{m}$ emission. For this to explain the trends we see in NGC 4565 and NGC 5907, they would need to suffer a factor of $\sim 2$ more diffuse $24 \mu \mathrm{m}$ contamination than the other galaxies whose slopes are consistent with that of NGC 4254 without diffuse emission (Rahman et al. 2011).

In order to check whether the difference between RADPROF (SFR) and PVD (CO) methods is responsible for the flatter slope in the SFR versus molecular gas relation, we can also investigate the relationship between $\Sigma_{\mathrm{SFR}}$ and $\Sigma_{\mathrm{H}_{2}}$ on a pixelby-pixel basis. For the pixel-by-pixel comparison, we use the masked CO intensity maps. Since most of the galaxies have very low resolution in $\mathrm{H}$ I compared to $\mathrm{CO}$, we do not attempt a pixel-by-pixel comparison between $\Sigma_{\mathrm{SFR}}$ and $\Sigma_{\text {gas }}$ and focus on the "molecular SFL" with the CO map convolved to the $24 \mu \mathrm{m}$ Gaussian beam (7") for NGC 4157, 4565, and 5907. The CO beam of NGC 891 is $7^{\prime \prime}$. Using the beam matched maps, we extract data on a pixel-by-pixel basis using the MIRIAD task IMCMP. All the extracted data are placed in 0.1 dex bins and the number of data points in each bin is mapped to the color scale in Figure 19. The blue solid points in the figures show the correlations from the radial profile analysis for comparison purposes. Most of the radial profile points for each galaxy follow the mean trends defined by the pixel-by-pixel plot (Figure 19), but their values are generally lower compared to the contours since the projection effect of an edge-on galaxy leads to higher values in the pixel-by-pixel method. In particular, NGC 4565 shows much lower values for the radial profile, due to its ring-like morphology leading to lower surface densities, especially in the central region. Note in our pixel-by- 

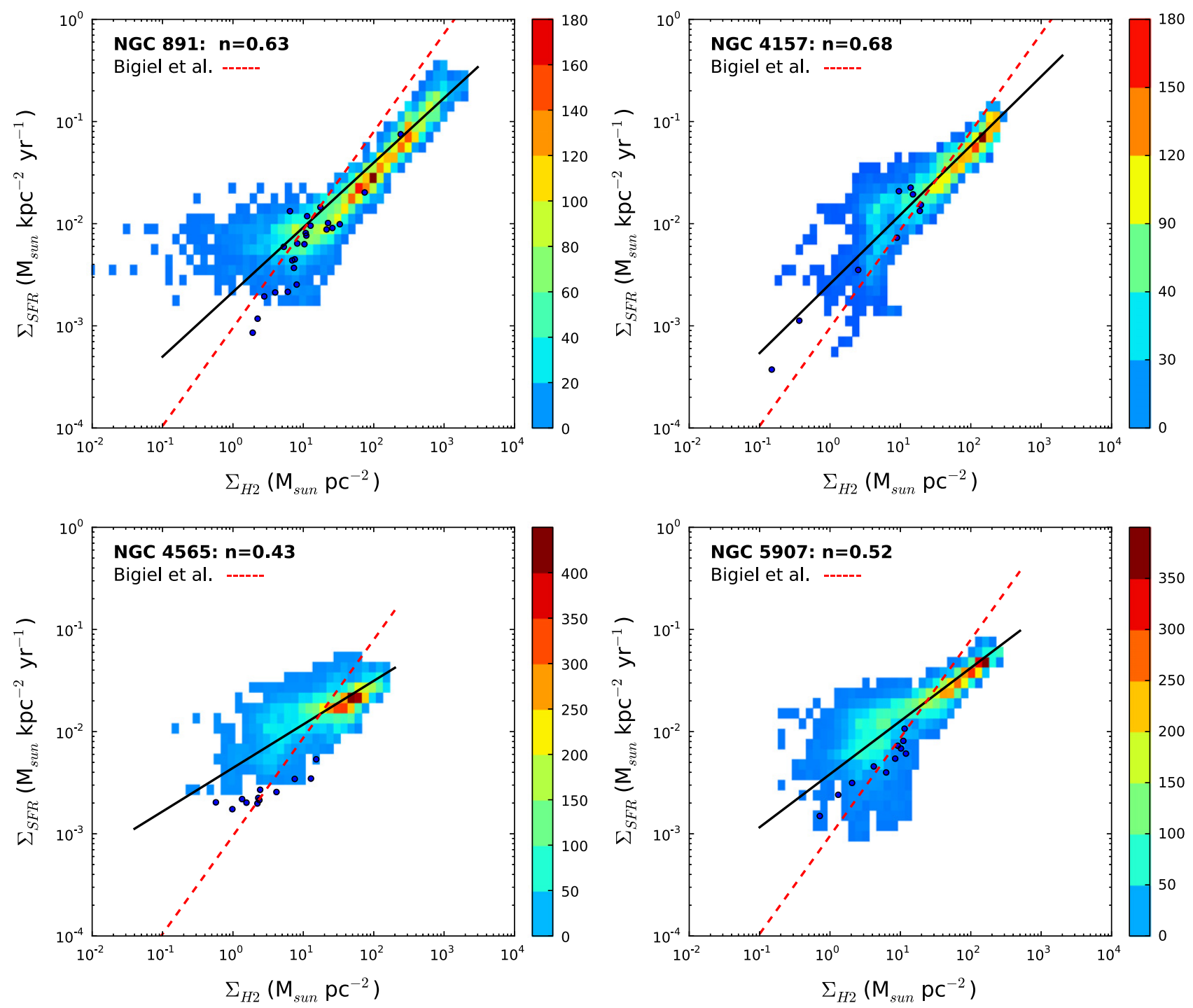

Figure 19. The relationship between $\Sigma_{\mathrm{SFR}}$ and $\Sigma_{\mathrm{H}_{2}}$ based on a pixel-by-pixel analysis. Color contours represent the numbers of the data points in a pixel (0.1 dex by $0.1 \mathrm{dex}$ ). The blue points show the relationship based on the radial profile shown in Figure 18 (left). The solid line is the best fit to the pixel-by-pixel data using the OLS bisector. The red dashed line shows the "molecular gas" Schmidt law index of 0.96 based on the pixel-by-pixel analysis of Bigiel et al. (2008).

(A color version of this figure is available in the online journal.)

pixel analysis, unlike most $\mathrm{K}-\mathrm{S}$ analyses, no deprojection is done.

The best-fit line in the figures is obtained using the OLS bisector and the fitted power-law index ( $\mathrm{K}-\mathrm{S}$ law index) is shown in the upper-left corner of the figure. The obtained indices from the pixel-by-pixel analysis are in the range of 0.43-0.68 for the molecular SFL. Those values are similar to the indices from the radial profile analysis. This is in contrast to the results of Bigiel et al. (2008) who obtained a power-law index of about unity (shown as red dashed line in Figure 19) for the $\mathrm{H}_{2}$ star formation relation based on a pixel-by-pixel comparison for seven spiral galaxies.

Figure 20 shows the star formation efficiency (SFE) as a function of radius (normalized by $R_{25}$ ) in terms of total gas $\left(\mathrm{SFE}=\Sigma_{\mathrm{SFR}} / \Sigma_{\text {gas }}\right)$ and the molecular gas $\left(\Sigma_{\mathrm{SFR}} / \Sigma_{\mathrm{H}_{2}}\right)$. The inverse of this quantity $\left(\mathrm{SFE}^{-1}\right)$ represents the gas depletion time. Overall, most galaxies show declining SFE (in terms of total gas) with radius even though fluctuations in SFE exist in some galaxies. On the other hand, the molecular SFE in Figure 20 (right) appears to increase with radius in some galaxies. This is caused by a steeper drop in the $\Sigma_{\mathrm{H}_{2}}$ profile compared to that of SFR and this situation could be due in part to the $24 \mu \mathrm{m}$ opacity and diffuse emission issues. Once again this contrasts with previous results which find a roughly constant SFE in the molecular gas. Rownd \& Young (1999) observed the constancy of SFE in a study of the SFE within CO-emitting galaxies. Bigiel et al. (2008) and Leroy et al. (2008) showed a constant SFE in the $\mathrm{H}_{2}$ dominated region and decreasing SFE with radius in the $\mathrm{H}_{\mathrm{I}}$ dominated region.

\subsection{Gravitational Instability}

Since both gas and stars can contribute to the gravitational instability of a galactic disk, many proposals have been made to evaluate stability using a single parameter $Q_{\text {tot }}$ analogous to the Toomre (1964) $Q$ parameter but including the properties of both gaseous and stellar disks (e.g., Jog \& Solomon 1984; Rafikov 2001; Romeo \& Wiegert 2011). A link between gravitational instability and star formation has been frequently suggested (Kennicutt 1989; Martin \& Kennicutt 2001; Li et al. 2005) but has been difficult to confirm observationally 

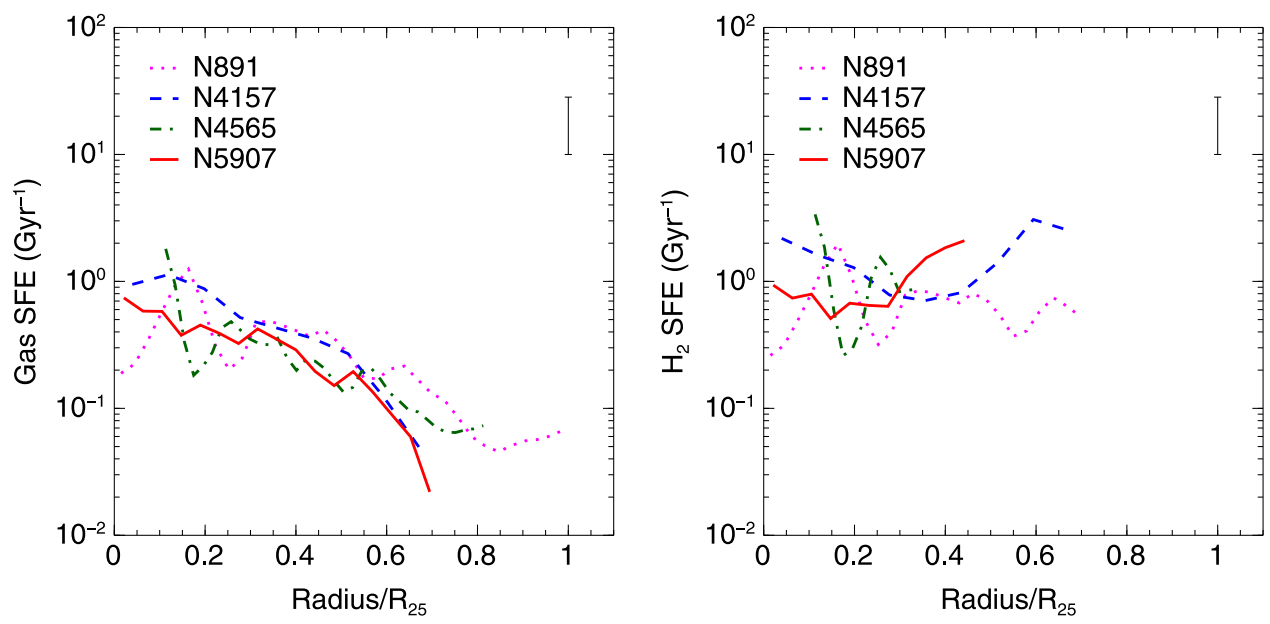

Figure 20. Star formation efficiency as a function of radius normalized by $R_{25}$ in terms of total gas (left) and the molecular gas (right) for NGC $891,4157,4565$, and 5907. The representative error bar of the SFE is shown in the upper right corner.

(A color version of this figure is available in the online journal.)
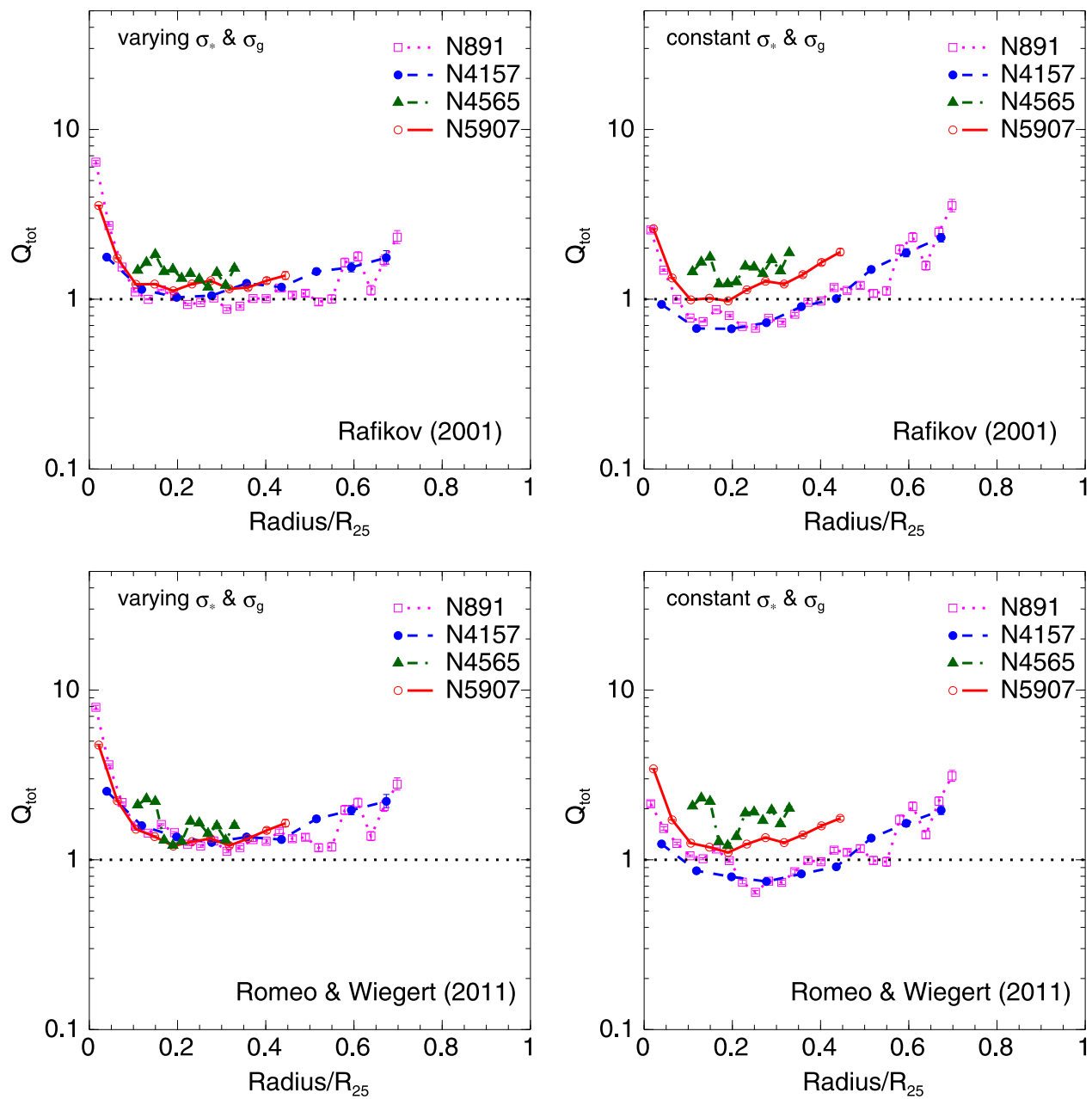

Figure 21. $Q_{\text {tot }}$ as a function of radius normalized by $R_{25}$ for varying velocity dispersions (left panels) and constant velocity dispersions (right panels). Unstable regions lie below the horizontal dotted lines. The top panels are obtained using $Q_{\text {tot }}$ provided by Rafikov (2001) and the bottom panels use the Romeo \& Wiegert (2011) approximation.

(A color version of this figure is available in the online journal.) 
(Wong \& Blitz 2002; Leroy et al. 2008). Recently, Elmegreen (2011) has suggested that $Q_{\text {tot }}$ primarily governs the spiral structure on large scales and has little direct influence on local collapse leading to star formation. He also suggests that gas dissipation can increase the stability threshold from $\sim 1$ to $2-3$ and allow small-scale instability to persist even at large $Q_{\text {tot }}$. In order to investigate the relationship between the gravitational instability parameter $Q$ and massive star formation, we obtain the parameter $Q_{\text {tot }}$ using a modified version (to treat $\mathrm{H}_{\mathrm{I}}$ and $\mathrm{H}_{2}$ separately) of the equation provided by Rafikov (2001), assuming two components of collisional gas and collisionless stars in a thin rotating galactic disk:

$$
\begin{aligned}
\frac{1}{Q_{\mathrm{tot}}}= & \frac{2}{Q_{\mathrm{HI}}} \Gamma_{\sigma_{\mathrm{HI}}} \frac{q}{1+q^{2} \Gamma_{\sigma_{\mathrm{HI}}}^{2}}+\frac{2}{Q_{\mathrm{H}_{2}}} \Gamma_{\sigma_{\mathrm{H}}} \frac{q}{1+q^{2} \Gamma_{\sigma_{\mathrm{H}}}^{2}} \\
& +\frac{2}{Q_{\mathrm{star}}} \frac{1}{q}\left[1-e^{-q^{2}} I_{0}\left(q^{2}\right)\right]>1,
\end{aligned}
$$

where

$$
\begin{gathered}
Q_{\mathrm{HI}}=\frac{\kappa \sigma_{\mathrm{HI}}}{\pi G \Sigma_{\mathrm{HI}}}, \quad Q_{\mathrm{H}_{2}}=\frac{\kappa \sigma_{\mathrm{H}_{2}}}{\pi G \Sigma_{\mathrm{H}_{2}}}, \\
Q_{\mathrm{star}}=\frac{\kappa \sigma_{*, R}}{\pi G \Sigma_{*}}, \\
\Gamma_{\sigma_{\mathrm{HI}}}=\sigma_{\mathrm{HI} / \sigma_{*, R},} \Gamma_{\sigma_{\mathrm{H} 2}}=\sigma_{\mathrm{H}_{2} /} \sigma_{*, R}, \\
q=\frac{k \sigma_{*, R}}{\kappa}, \quad \kappa=\frac{V_{\mathrm{rot}}}{R} \sqrt{2\left(1+\frac{R}{V_{\mathrm{rot}}} \frac{d V_{\mathrm{rot}}}{d R}\right) .}
\end{gathered}
$$

Here $\sigma_{*, R}$ is the stellar velocity dispersion in the radial direction, estimated from the vertical velocity dispersion: $\sigma_{*, R}=\sigma_{*} / 0.6$ (Bottema 1993). $\mathrm{I}_{0}$ is the Bessel function of zero order, $k$ is the wavenumber $(2 \pi / \lambda), \kappa$ is the epicyclic frequency, and $V_{\text {rot }}$ is the rotational velocity, obtained using Equation (2) with a correction for the inclination: $V_{\text {rot }}=V_{\text {proj }} / \sin i$. Note that $Q_{\text {tot }}$ is the minimum among those values we can obtain with a range of $\lambda$; usually the selected $\lambda$ (providing the minimum $Q_{\text {tot }}$ ) is up to about $4 \mathrm{kpc}$. In this procedure, we use the inferred vertical velocity dispersion as a function of radius (Figure 16) to derive the parameter $Q_{\text {tot }}$ shown in the left panel of Figure 21 for the galaxies including NGC 891 from Paper I. The error bars of $Q_{\text {tot }}$ show maximum and minimum values implied by the uncertainties in $\Sigma_{\mathrm{H}_{2}}, \Sigma_{\mathrm{HI}}$, and $\Sigma_{*}$. Since the differences between the maximum and minimum values are very small, the error bars are not very noticeable. The unstable condition is $Q_{\mathrm{tot}}<1$. The results show that most galaxies are marginally stable $(Q \sim 1)$ in most regions, supporting the self-regulation of star formation (e.g., Ostriker et al. 2010; Kim et al. 2011). The $Q_{\text {tot }}$ value is increasing toward the center, contradicting the general expectation of an unstable central region. This tendency is also shown in a study of Leroy et al. (2008) even though they used a constant velocity dispersion for gas $\left(11 \mathrm{~km} \mathrm{~s}^{-1}\right)$ and varying velocity dispersion of stars proportional to $\Sigma_{*}^{0.5}$. The increase of $Q$ toward the center appears to be due to the epicyclic frequency associated with the steeply rising slope of the rotation curve. It is well known that a strong central mass concentration makes $Q$ increase in the central regions; this is called the " $Q$ barrier" and is discussed by Sellwood (1985) and others. Generally, the $Q_{\text {tot }}$ profiles beyond the central regions are consistent with the total gas SFE; $Q$ is rising as the SFE decreases.

In Figure 21 (top right), we obtain another $Q_{\text {tot }}$ profile with the vertical velocity dispersions assumed to be constant $\left(\sigma_{*}\right.$ $=21 \mathrm{~km} \mathrm{~s}^{-1}$ and $\sigma_{\mathrm{g}}=8 \mathrm{~km} \mathrm{~s}^{-1}$ ), which is commonly assumed in the literature (e.g., Combes \& Becquaert 1997; Rafikov 2001). In this regime, two galaxies (NGC 891 and 4157) show unstable regions predicting star formation in the inner disk, while the other galaxies (NGC 4565 and 5907) show a marginally stable disk. Considering the relatively low SFR in NGC 4565 and 5907 compared to the SFR in NGC 891 and 4157 , the $Q_{\text {tot }}$ profile with constant velocity dispersions shows a reasonable prediction for the massive star formation. However, the assumed constant values are in conflict with the inferred radial variation in the velocity dispersion. The increase toward the center is also shown in this profile.

The different $Q_{\text {tot }}$ profiles for constant and varying velocity dispersions show how much the adopted velocity dispersions affect the parameter. The variable velocity dispersions bring the $Q$ versus $R$ trends into better agreement galaxy-to-galaxy. By plotting individual $Q$ profiles for gas and stars in both cases, it appears that $Q_{\text {tot }}$ is affected mostly by $Q_{\text {star }}$. The range and values of $Q_{\text {gas }}$ are very large compared to $Q_{\text {star }}$ and the profile shape of $Q_{\text {tot }}$ follows that of $Q_{\text {star }}$. One can see the individual $Q$ profiles in Paper I (Figure 15) for NGC 891.

For comparison purposes, we also applied the recent approximation considering realistically thick disks given by Romeo \& Wiegert (2011):

$$
\frac{1}{Q_{\mathrm{tot}}}=\left\{\begin{array}{l}
\frac{W}{T_{*} Q_{\mathrm{star}}}+\frac{1}{T_{\mathrm{g}} Q_{\mathrm{gas}}} \text { if } T_{*} Q_{\mathrm{star}} \geqslant T_{\mathrm{g}} Q_{\mathrm{gas}}, \\
\frac{1}{T_{*} Q_{\mathrm{star}}}+\frac{W}{T_{\mathrm{g}} Q_{\mathrm{gas}}} \text { if } T_{\mathrm{g}} Q_{\mathrm{gas}} \geqslant T_{*} Q_{\mathrm{star}},
\end{array}\right.
$$

where

$$
W=\frac{2 s}{1+s^{2}}, \quad s=\frac{\sigma_{\mathrm{g}}}{\sigma_{*, R}}, \quad T \approx 0.8+0.7\left(\frac{\sigma_{z}}{\sigma_{R}}\right) .
$$

Here the ratio of vertical to radial velocity dispersion $\left(\sigma_{z} / \sigma_{R}\right)$ is 0.6 for stars and 1 for gas. The $Q_{\text {tot }}$ profiles using varying and constant $\sigma$ are shown in the bottom panels of Figure 21. Even though there is not much difference between the two different approaches provided by Rafikov (2001) and Romeo \& Wiegert (2011), the Rafikov $Q_{\text {tot }}$ values using varying $\sigma$ seem consistently lower than the Romeo and Wiegert values by enough that the Rafikov values are $\sim 1$ but the Romeo and Wiegert values are usually $\geqslant 1$. Overall, they show similar behavior in the profiles although the values are slightly different.

\section{MOLECULAR TO ATOMIC GAS RATIO}

The ratio of molecular to atomic gas density is expected to scale with the interstellar gas pressure (e.g., Elmegreen 1993). Since SFR is strongly correlated with the molecular gas, the interstellar pressure may provide a prescription for the SFR in a galaxy, if there is a strong correlation between the interstellar pressure and the ratio. Therefore, we examine whether the correlation is applicable in our sample of galaxies. First, we have investigated the relationship between the hydrostatic midplane pressure and the molecular to atomic gas surface density ratio $\left(\Sigma_{\mathrm{H}_{2}} / \Sigma_{\mathrm{HI}}\right)$. In order to obtain the hydrostatic 

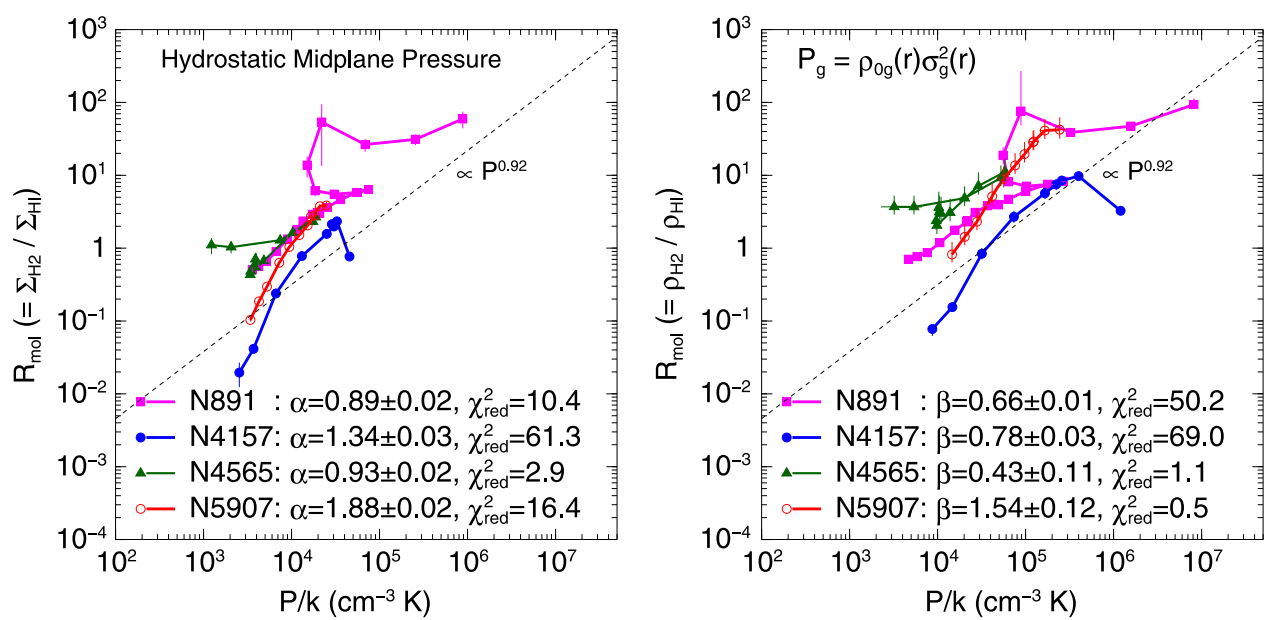

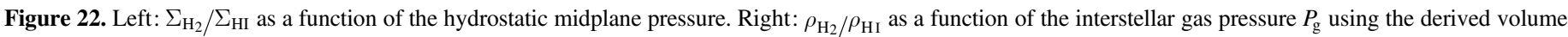

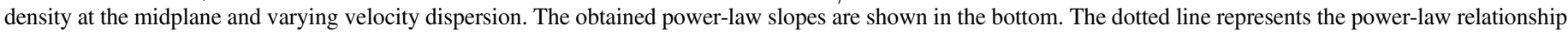
with slope of 0.92 from Blitz \& Rosolowsky (2006) for comparison.

(A color version of this figure is available in the online journal.)

pressure $\left(\mathrm{P}_{0}\right)$, we use the equation given by Paper I:

$$
P_{0}=0.89\left(G \Sigma_{*}\right)^{0.5} \sum_{\text {gas }} \frac{\sigma_{\mathrm{g}}}{z_{*}^{0.5}} .
$$

This equation is very close to that derived by Blitz \& Rosolowsky (2004). We assume the gas velocity dispersion $\sigma_{\mathrm{g}}=8 \mathrm{~km} \mathrm{~s}^{-1}$ following Blitz \& Rosolowsky (2004). For the constant stellar scale height $\left(z_{*}\right)$, the values obtained by fitting the exponential model to $3.6 \mu \mathrm{m}$ maps in Section 3.2 are used: $780 \mathrm{pc}, 640 \mathrm{pc}$, and $670 \mathrm{pc}$ for NGC 4157, 4565, and 5907, respectively. In order to compare with the power-law relationship found by Blitz \& Rosolowsky (2006), we plot the ratio $\Sigma_{\mathrm{H}_{2}} / \Sigma_{\mathrm{HI}}$ against the hydrostatic pressure in Figure 22 (left panel) and obtain a power-law slope $(\alpha)$ by least-squares fitting:

$$
\frac{\Sigma_{\mathrm{H}_{2}}}{\Sigma_{\mathrm{HI}}}=\left(\frac{P_{0}}{P_{\mathrm{tr}}}\right)^{\alpha},
$$

where $P_{\text {tr }}$ is the hydrostatic pressure at the transition radius where $\Sigma_{\mathrm{H}_{2}} / \Sigma_{\mathrm{HI}}=1$ in the fit. The obtained power-law indices and reduced $\chi^{2}$ (goodness of fit) values are shown in the bottom of the figure. The dotted line in the figure represents a power-law relationship with index of 0.92 as found by Blitz \& Rosolowsky (2006) for comparison. It seems that most of galaxies are well fitted by the power-law relation even though the range of slopes is large. However, the index of 0.92 is an average value and the range of slopes for the sample of galaxies in the study by Blitz \& Rosolowsky (2006) is $0.58-1.64$. The relation in two galaxies (NGC 4157 and 5907) appears to steepen in the outer disk due to the rapid decrease in $\Sigma_{\mathrm{H}_{2}}$ in the region.

The midplane hydrostatic pressure $\left(P_{0}\right)$ is obtained by assuming constant values for the stellar scale height and gaseous velocity dispersion. However, those values vary with radius as shown in Figures 13 and 16. Therefore, we have derived the interstellar gas pressure using the derived volume density at the midplane and radially dependent vertical velocity dispersion in order to examine whether a power-law relationship is still valid for the interstellar gas pressure $\left(P_{\mathrm{g}}\right)$ at the midplane:

$$
P_{\mathrm{g}}=\rho_{0 \mathrm{~g}}(R) \sigma_{\mathrm{g}}^{2}(R) \approx \rho_{0 \mathrm{H}_{2}}(R) \sigma_{\mathrm{H}_{2}}^{2}(R)+\rho_{0 \mathrm{HI}}(R) \sigma_{\mathrm{HI}}^{2}(R) .
$$

We assume the partial pressures contributed by the $\mathrm{H}_{\mathrm{I}}$ and $\mathrm{H}_{2}$ are additive. We find that a power-law relationship between the midplane $\rho_{\mathrm{OH}_{2}} / \rho_{\mathrm{OHI}}$ ratio and the interstellar pressure seems to roughly hold as shown by Figure 22 (right panel). The vertical and horizontal error bars in Figure 22 show uncertainties in $R_{\mathrm{mol}}$ and pressure based on maximum and minimum values for volume density and velocity dispersion that were estimated from uncertainties in the surface densities and scale heights. In addition, the uncertainty (a factor of 2) of the CO-to- $\mathrm{H}_{2}$ conversion factor would affect the pressure using varying $\sigma_{\mathrm{g}}(R)$ by a factor of $\sim 20 \%$ for NGC $4157, \sim 30 \%$ for NGC 4565 , and $\sim 25 \%$ for NGC 5907. The power-law indices are a bit smaller compared with using constant values for $\sigma_{\mathrm{g}}$ and $z_{*}$ (left panel) but remain in good agreement with Blitz \& Rosolowsky (2006). The reason why the relation based on volume density and varying velocity dispersion still shows a good correlation is that both $R_{\mathrm{mol}}$ and $P$ are shifted to higher values: $R_{\mathrm{mol}}$ is higher at the midplane because the $\mathrm{CO}$ disk is thinner than the $\mathrm{H} \mathrm{I}$, but the midplane pressure is also higher since the inferred velocity dispersion is much higher than the assumed value of $8 \mathrm{~km} \mathrm{~s}^{-1}$. The two effects together tend to move the points up and to the right, so the correlation is roughly preserved. However, as noted in Paper I, the correlation is not preserved out of the midplane (at least for NGC 891; see Figure 17 of that paper).

\section{SUMMARY AND CONCLUSIONS}

We have measured the thicknesses of $\mathrm{CO}, \mathrm{HI}$, and stellar disk for a sample of edge-on galaxies (NGC 4157, 4565, and 5907) in order to derive the midplane volume density as a function of radius. In addition, we have inferred the vertical velocity dispersion from the measured disk thickness and surface density by solving the Poisson equation. Using the estimated volume density and inferred velocity dispersion as functions of radius, we have derived the interstellar gas pressure $\left(\rho_{0 \mathrm{~g}} \sigma_{\mathrm{g}}^{2}\right)$ in order to investigate the role of the pressure 

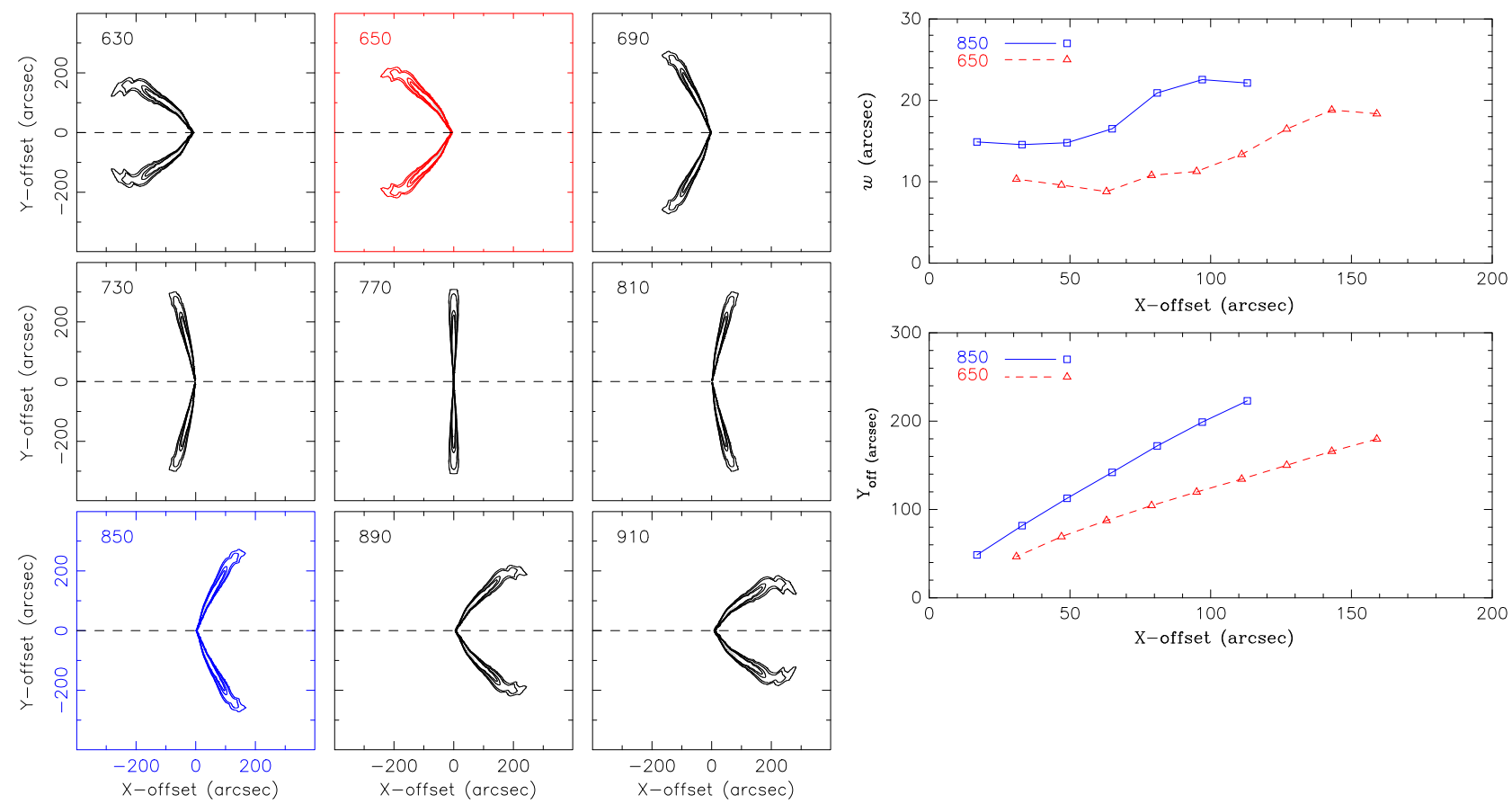

Figure A1. Left: deprojected channel maps of a model galaxy generated using the velocity field map, assumed velocity dispersion of $8 \mathrm{~km} \mathrm{~s}^{-1}$, and the $\mathrm{H}_{\mathrm{I}}$ surface density profile of NGC 4157. The radial velocity of each channel map is indicated in the upper left corner. The systemic velocity is $770 \mathrm{~km} \mathrm{~s}{ }^{-1}$. Right top: Gaussian width $(w)$ against $x$-offset from the channel maps at 650 and $850 \mathrm{~km} \mathrm{~s}^{-1}$. Right bottom: minor axis offset from the midplane $\left(y_{\text {off }}\right)$ as a function of $x$-offset from the channel maps at 650 and $850 \mathrm{~km} \mathrm{~s}^{-1}$.

(A color version of this figure is available in the online journal.)
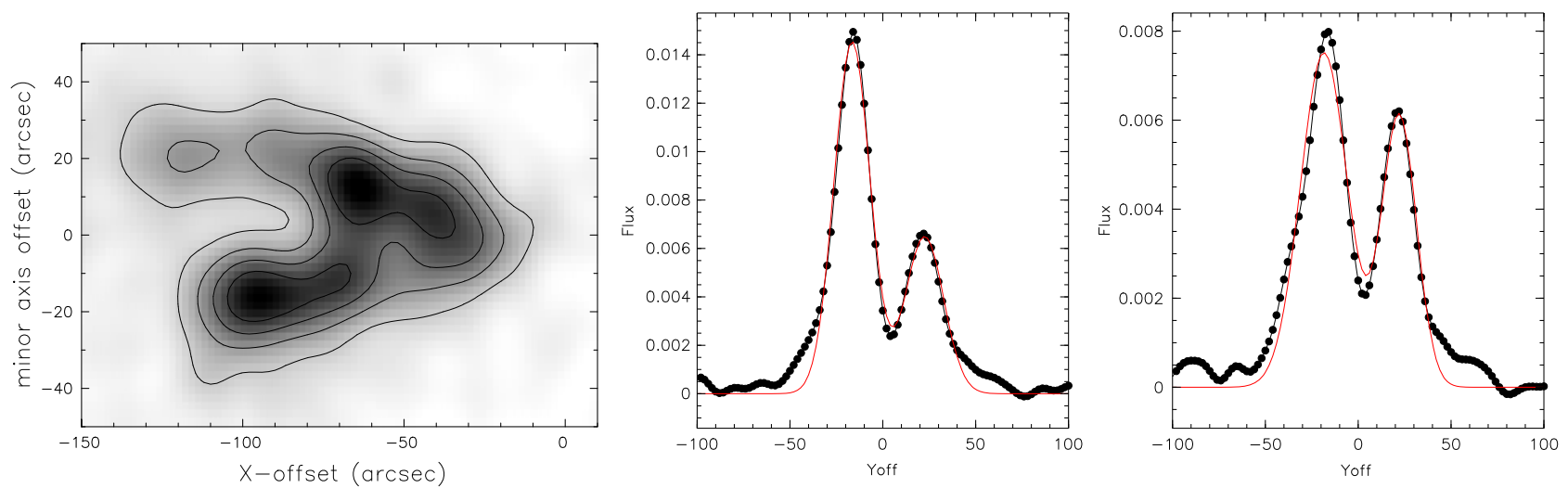

Figure A2. Velocity channel map (taken at $V_{R}=670 \mathrm{~km} \mathrm{~s}^{-1}$ ) for the NGC $4157 \mathrm{H} \mathrm{I}$ data (left). Double Gaussian fit (red line) to the minor axis profile (black line) at $x=-95^{\prime \prime}$ (middle) and at $x=-111^{\prime \prime}$ (right).

(A color version of this figure is available in the online journal.)

in controlling the $\mathrm{H}_{2} / \mathrm{H}_{\text {I }}$ ratio. Our conclusions are summarized below.

1. We have obtained the inclinations using the Olling (1996) method to consider the projection effect of the less edge-on galaxies when we derive the disk thickness. The measured inclinations of NGC 4157 show a small difference between $\mathrm{CO}\left(84^{\circ}\right)$ and $\mathrm{HI}\left(83^{\circ}\right)$ disks, while NGC 4565 and 5907 show a consistent value for both disks: $86.5^{\circ}$ for NGC 4565 and $86^{\circ}$ for NGC 5907.

2. Using the inclinations, we have derived the gas disk thickness from the FWHM values of the observational data and the model at many different channel maps showing a "butterfly" shape in the Appendix. Our results show that the scale heights of $\mathrm{CO}, \mathrm{HI}$, and stars increase with radius over the disk although the flaring of the $\mathrm{CO}$ disk in NGC 4565 and 5907 is not clearly visible. The strongly star-forming galaxies (NGC 891 and NGC 4157) show a thicker CO disk compared to the other galaxies, suggesting a correlation between the disk thickness and star formation activity. The vertical velocity dispersions of $\mathrm{CO}, \mathrm{H}_{\mathrm{I}}$, and stars inferred using the scale height and the surface density are declining as a function of radius.

3. We have obtained the Schmidt law index for the total gas and the molecular gas using both the radial profile and the pixel-by-pixel analyses. For the molecular SFL, the fitted indices for all galaxies range from 0.32 to 0.91 in the radial profile method and from 0.43 to 0.68 in the pixel-by-pixel method. For the total gas SFL, the range of the index is from 1.42 to 3.41 in the radial profile analysis. The 


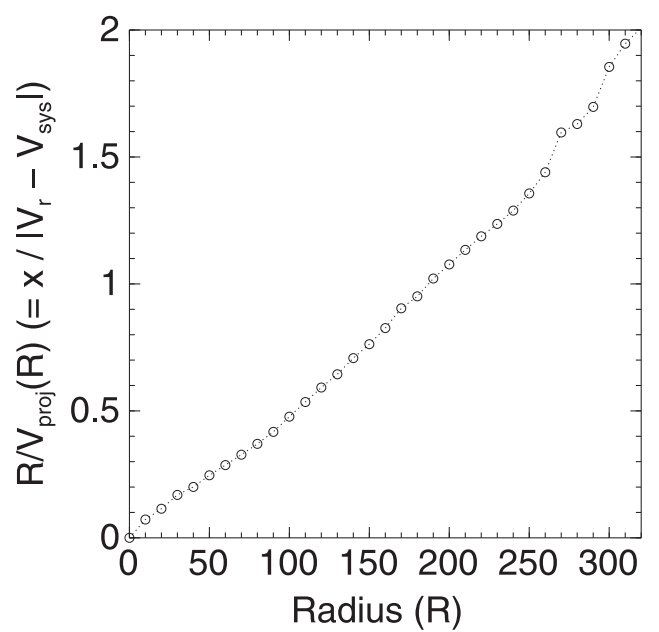

Figure A3. $R / V_{\text {proj }}(R)\left(=x /\left|V_{R}-V_{\text {sys }}\right|\right)$ against radius for NGC 4157 .

correlation between $\Sigma_{\mathrm{SFR}}$ and $\Sigma_{\mathrm{H}_{2}}$ appears to be stronger than the relationship between $\Sigma_{\text {SFR }}$ and $\Sigma_{\text {gas }}$ when taking into account the rms scatter and the curvature in the relationship for the total gas.

4. We have derived the gravitational instability parameter $Q_{\text {tot }}$ in order to investigate the relationship between the parameter and massive star formation. In addition, we have compared the $Q_{\text {tot }}$ profiles using constant and varying velocity dispersions of gas and stars. Our results show that $Q_{\text {tot }}$ using the constant velocity dispersions seems to predict the star-forming region in the inner disk, while $Q_{\text {tot }}$ using the varying velocity dispersions does not show a clear relationship with massive star formation. In addition, both cases are not able to predict massive star formation near the center. While the gravitational instability parameter does not appear to predict the locus of massive star formation, the parameter using the varying velocity dispersions is suggestive of self-regulated star formation $(Q \sim 1)$ even though the values are a bit larger than unity in most of galaxies.

5. We have obtained the power-law index of the relationship between the hydrostatic midplane pressure and the molecular to atomic gas surface density ratio. The range of the fitted index for the galaxies in this study is 0.89-1.88. In addition, we have derived the interstellar gas pressure using the derived volume density and varying velocity dispersion $\sigma_{\mathrm{g}}(R)$ in order to examine the powerlaw relationship between the gas pressure and the ratio of $\mathrm{H}_{2}$ to $\mathrm{H}_{\mathrm{I}}$ density at the midplane $\left(\rho_{\mathrm{OH}_{2}} / \rho_{\mathrm{OHI}}\right)$. The powerlaw correlation between the pressure and the ratio is still valid at the midplane even if we use the varying scale heights and velocity dispersions. The power-law index for the relationship based on $\sigma_{\mathrm{g}}(R)$ ranges from 0.43 to 1.54 .

We thank the anonymous referee for useful comments and suggestions that improved this paper. K.Y. thanks Laura Zschaechner and Gyula Józsa for help with using TiRiFiC. This study is supported by the National Science Foundation under cooperative agreements AST-0838226 and AST-1139950 and by a Spitzer Cycle-5 data analysis award from NASA. Support for CARMA construction was derived from the states of California, Illinois, and Maryland, the James S. McDonnell Foundation, the Gordon and Betty Moore Foundation, the
Kenneth T. and Eileen L. Norris Foundation, the University of Chicago, the Associates of the California Institute of Technology, and the National Science Foundation. Ongoing CARMA development and operations are supported by the National Science Foundation under a cooperative agreement, and by the CARMA partner universities. K.Y. and J.M.H. acknowledge support from the European Research Council under the European Union's Seventh Framework Programme (FP/2007-2013) / ERC Grant Agreement No. 291531. The National Radio Astronomy Observatory is a facility of the National Science Foundation operated under cooperative agreement by Associated Universities, Inc.

\section{APPENDIX \\ INCLINATION AND SCALE HEIGHT USING OLLING'S METHOD}

In order to derive the inclination and the scale height for less edge-on galaxies, we have employed the method by Olling (1996) and we describe it as follows. The inclination $(i)$ and the disk thickness (FWHM) are obtained from the apparent disk width $\left(\mathrm{FWHM}_{\mathrm{obs}}\right)$ measured at each velocity by taking into account the size of the in-plane region that contributes emission at that velocity.

First, we have created model emission cubes using the rotation curve (Figure 8) that we derived in Section 3.1, the surface density profile (Figure 9), and an assumed constant velocity dispersion $\left(8 \mathrm{~km} \mathrm{~s}^{-1}\right)$ for both $\mathrm{CO}$ and $\mathrm{HI}$ maps. For NGC 4157 and 5907, we use the CO rotation curve (red dashed line in Figure 8) for the $\mathrm{CO}$ model and the $\mathrm{H}$ I rotation curve (blue solid line in the figure) for the $\mathrm{H}$ i model. However, in the case of NGC 4565, we use the $\mathrm{H}$ i rotation curve for both $\mathrm{CO}$ and $\mathrm{H}_{\mathrm{I}}$ models because of a lack of $\mathrm{CO}$ emission in the inner region of the galaxy. Since the rotation curves of $\mathrm{CO}$ and $\mathrm{H}_{\mathrm{I}}$ in this galaxy are very consistent, using the $\mathrm{H}_{\mathrm{I}}$ curve for the $\mathrm{CO}$ model is justified.

In this procedure, we used a modified version of the MIRIAD task VELMODEL to produce a model of the in-plane distribution of gas at each observed radial velocity. From this task, we obtain a face-on view of the emission contributing to each observed velocity channel, using the rotation curve and the systemic velocity. The structure of the velocity field depends on the adopted rotation curve. In addition, we used the MIRIAD task IMGEN to make a velocity dispersion model (assuming $\sigma_{\mathrm{g}}=8 \mathrm{~km} \mathrm{~s}^{-1}$ ) and a modified version of the task ELLINT to build a surface density model (using our derived surface density profile). For combining all three one-dimensional models (velocity field, velocity dispersion, and surface density models) together to make the model cube, the task VELIMAGE has been employed.

Second, we have fitted a double Gaussian profile $\left(e^{-0.5\left(\left(y-y_{\text {off }}\right) / w\right)^{2}}+e^{-0.5\left(\left(y+y_{\text {off }}\right) / w\right)^{2}}\right)$ to the minor axis distribution at each $x$-offset for a given channel map of the model cube in order to obtain the Gaussian width $(w)$ and the vertical offset $\left(y_{\text {off }}\right)$ from the line of nodes $(y=0)$. Some channel maps of the model are shown in Figure A1 (left) and the Gaussian width and the vertical offset from the midplane obtained from the velocity channel map of $650 \mathrm{~km} \mathrm{~s}^{-1}$ and $850 \mathrm{~km} \mathrm{~s}^{-1}$ are shown in the figure (right). The planar width $\left(\mathrm{FWHM}_{\mathrm{p}}\right)$ of the model 

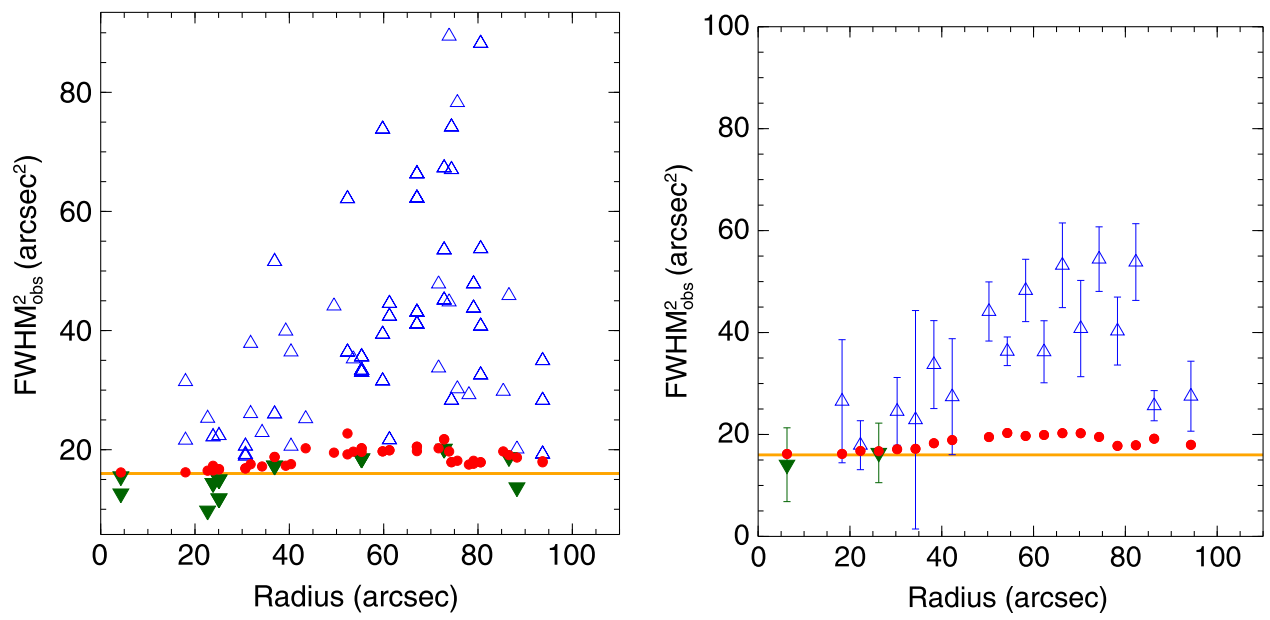

Figure A4. Left: the observed FWHM values (open blue and solid green triangles) and the sum of $\mathrm{FWHM}_{\mathrm{beam}}^{2}$ and $\mathrm{FWHM}_{\mathrm{p}}^{2} \cos ^{2} i$ (solid red circle) as a function of radius for $\mathrm{CO}$ in NGC 4157. The inverted green triangles show the non-deconvolveable data since they are below the values of the solid circles. Right: averaged data points in $4^{\prime \prime}$ radial bins. The values are for valid double Gaussian fits. The inverted triangle points show the data, which are non-deconvolveable. The horizontal line represents a value of FWHM $_{\text {beam }}^{2}$.

(A color version of this figure is available in the online journal.)

is given by

$$
\operatorname{FWHM}_{\mathrm{p}}\left(x, V_{R}\right) \approx 2.35 \sqrt{w^{2}\left(x, V_{R}\right)+y_{\text {off }}^{2}\left(x, V_{R}\right)},
$$

where the minor axis offset $y_{\text {off }}$ is ignored when the minor axis profile can be fit with two Gaussians.

Third, returning to the observed data cubes, we have determined distances between the two peaks of the minor axis profile $(\Delta d)$ and the $\mathrm{FWHM}_{\mathrm{obs}}$ thicknesses $(2.35 \times$ Gaussian width) as functions of $x$ and the radial velocity $\left(V_{R}\right)$ by fitting a double Gaussian profile to many velocity channel maps. The channel maps of observational data are selected when they show clear a "butterfly" shape, so they can be fitted by a double Gaussian profile. In addition, among many fitted $x$-values in the selected channel map, the final parameters we use for deriving the inclination and the disk thickness are chosen when at least one of two peaks is greater than twice the rms noise of the channel map and $\Delta d$ is greater than the synthesized beam $\left(\mathrm{FWHM}_{\text {beam }}\right)$. One of the channel maps we have used for fitting is shown in Figure A2 and examples of fitting results at two different $x$ offsets in the channel map are also shown in the figure.

Finally, using all the parameters we obtained above, we have derived inclinations $(i)$ and the disk thicknesses as a function of radius for the galaxies using the equations given by Olling (1996):

$$
\begin{aligned}
i\left(x, V_{R}\right) & =\cos ^{-1} \frac{\Delta d\left(x, V_{R}\right)}{2 y_{\text {off }}\left(x, V_{R}\right)}, \\
\operatorname{FWHM}^{2}\left(x, V_{R}\right)= & \frac{1}{\sin ^{2} i}\left\{\operatorname{FWHM}_{\mathrm{obs}}^{2}\left(x, V_{R}\right)-\mathrm{FWHM}_{\text {beam }}^{2}\right. \\
& \left.-\left[\mathrm{FWHM}_{\mathrm{p}}\left(x, V_{R}\right) \cos i\right]^{2}\right\}
\end{aligned}
$$

where $\mathrm{FWHM}_{\text {beam }}$ is beam size of the maps after convolving to the circular beam shown in Table 2. One double Gaussian fit to the butterfly shape of the data provides two different values of FWHM since the Gaussian widths from both sides are independent, unlike the widths of the model. We use both FWHM values when the ratio of smaller to larger peak intensities is more than a factor of 0.6, while just one FWHM having the higher peak value is used when the peak ratio is less than 0.6. We convert $x$ to a radius $(R)$ using the projected rotation curve $\left(V_{\text {proj }}\right)$, which is used to obtain the velocity field model:

$$
R=V_{\mathrm{proj}}(R) \frac{x}{\left|V_{R}-V_{\mathrm{sys}}\right|} .
$$

We plot $R / V_{\text {proj }}(R)\left(=x /\left|V_{R}-V_{\text {sys }}\right|\right)$ versus radius $(R)$ in Figure A3, showing how we obtain a radius corresponding to each combination of $\left(x, V_{R}\right)$. From the inclination as a function of radius, we derived a weighted mean value of inclination and fixed the inclination to this value when deriving the FWHM values.

As an example, the input needed to obtain FWHM thickness values are shown in Figure A4 (left panel) for the CO image of NGC 4157. The open triangles show the observed width $\left(\mathrm{FWHM}_{\mathrm{obs}}^{2}\right)$ and filled circles represent the sum of the two broadening terms $\left(\mathrm{FWHM}_{\text {beam }}^{2}+\mathrm{FWHM}_{p}^{2} \cos ^{2} i\right)$ in Equation (A3). In the right panel, all the points are averaged in a $4^{\prime \prime}$ radial bin and the averaged values are used to derive the FWHM thickness following Equation (A3). The inverted triangle points show the data, which are non-deconvolvable since they are below the values of the solid circles. The bin size is selected based on the angular resolution of each map: $4^{\prime \prime}$ for all the CO maps, $10^{\prime \prime}$ for the H I map of NGC 4565 , and $15^{\prime \prime}$ for the Hi maps of NGC 4157 and 5907. The use of an average value in a bin provides a resolution similar to its map, as well as a better signal-to-noise ratio, hence we adopt this approach for our analysis.

\section{REFERENCES}

Aniano, G., Draine, B. T., Gordon, K. D., \& Sandstrom, K. 2011, PASP, 123, 1218

Bigiel, F., Leroy, A., Walter, F., et al. 2008, AJ, 136, 2846

Blitz, L., \& Rosolowsky, E. 2004, ApJL, 612, L29

Blitz, L., \& Rosolowsky, E. 2006, ApJL, 650, L933

Bohlin, R. C., Savage, B. D., \& Drake, J. F. 1978, ApJ, 224, 132

Bolatto, A. D., Wolfire, M., \& Leroy, A. K. 2013, ARA\&A, 51, 207

Bottema, R. 1993, A\&A, 275, 16 
Calzetti, D., Kennicutt, R. C., Engelbracht, C. W., et al. 2007, ApJ, 666, 870 Combes, F., \& Becquaert, J.-F. 1997, A\&A, 326, 554

Dahlem, M., Ehle, M., Ryder, S. D., Vlajić, M., \& Haynes, R. F. 2005, A\&A, 432,475

Dame, T. M., Hartmann, D., \& Thaddeus, P. 2001, ApJ, 547, 792

de Grijs, R., \& Peletier, R. F. 1997, A\&A, 320, L21

Dumke, M., Braine, J., Krause, M., et al. 1997, A\&A, 325, 124

Elmegreen, B. G. 1993, ApJ, 411, 170

Elmegreen, B. G. 2011, ApJ, 737, 10

Ferguson, A. M. N., Wyse, R. F. G., Gallagher, J. S., \& Hunter, D. A. 1998 , ApJL, 506, L19

Garcia-Burillo, S., Guelin, M., \& Neininger, N. 1997, A\&A, 319, 450

Indebetouw, R., Mathis, J. S., Babler, B. L., et al. 2005, ApJ, 619, 931

Irwin, J. A., English, J., \& Sorathia, B. 1999, AJ, 117, 2102

Isobe, T., Feigelson, E. D., Akritas, M. G., \& Babu, G. J. 1990, ApJ, 364, 104

Jog, C. J., \& Solomon, P. M. 1984, ApJ, 276, 114

Józsa, G. I. G., Kenn, F., Klein, U., \& Oosterloo, T. A. 2007, A\&A, 468, 731

Just, A., Möllenhoff, C., \& Borch, A. 2006, A\&A, 459, 703

Kennicutt, R. C. Jr. 1989, ApJ, 344, 685

Kennicutt, R. C. Jr. 1998, ApJ, 498, 541

Kim, C.-G., Kim, W.-T., \& Ostriker, E. C. 2011, ApJ, 743, 25

Krumholz, M. R., Dekel, A., \& McKee, C. F. 2012, ApJ, 745, 69

Laine, S., Appleton, P. N., Gottesman, S. T., Ashby, M. L. N., \& Garland, C. A. 2010, AJ, 140, 753

Leroy, A., Bolatto, A. D., Simon, J. D., \& Blitz, L. 2005, ApJ, 625, 763

Leroy, A. K., Walter, F., Brinks, E., et al. 2008, AJ, 136, 2782

Li, Y., Mac Low, M.-M., \& Klessen, R. S. 2005, ApJL, 620, L19

Martin, C. L., \& Kennicutt, R. C. Jr. 2001, ApJ, 555, 301

McMullin, J. P., Waters, B., Schiebel, D., Young, W., \& Golap, K. 2007 in ASP Conf. Ser. 376, Astronomical Data Analysis Software and Systems XVI, ed. R. A. Shaw, F. Hill, \& D. J. Bell (San Francisco, CA: ASP), 127 Murphy, E. J., Bremseth, J., Mason, B. S., et al. 2012, ApJ, 761, 97

Narayan, C. A., \& Jog, C. J. 2002, A\&A, 394, 89
O’Brien, J. C., Freeman, K. C., \& van der Kruit, P. C. 2010, A\&A, 515, A62

Olling, R. P. 1996, AJ, 112, 457

Oosterloo, T., Fraternali, F., \& Sancisi, R. 2007, AJ, 134, 1019

Ostriker, E. C., McKee, C. F., \& Leroy, A. K. 2010, ApJ, 721, 975

Petric, A. O., \& Rupen, M. P. 2007, AJ, 134, 1952

Rafikov, R. R. 2001, MNRAS, 323, 445

Rahman, N., Bolatto, A. D., Wong, T., et al. 2011, ApJ, 730, 72

Rahman, N., Bolatto, A. D., Xue, R., et al. 2012, ApJ, 745, 183

Rieke, G. H., \& Lebofsky, M. J. 1985, ApJ, 288, 618

Romeo, A. B., \& Wiegert, J. 2011, MNRAS, 416, 1191

Rownd, B. K., \& Young, J. S. 1999, AJ, 118, 670

Rupen, M. P. 1991, AJ, 102, 48

Sancisi, R. 1976, A\&A, 53, 159

Sanders, D. B., Mazzarella, J. M., Kim, D.-C., Surace, J. A., \& Soifer, B. T. 2003, AJ, 126, 1607

Schmidt, M. 1959, ApJ, 129, 243

Sellwood, J. A. 1985, MNRAS, 217, 127

Sheth, K., Regan, M., Hinz, J. L., et al. 2010, PASP, 122, 1397

Sofue, Y. 1996, ApJ, 458, 120

Strong, A. W., \& Mattox, J. R. 1996, A\&A, 308, L21

Tamburro, D., Rix, H., Leroy, A. K., et al. 2009, AJ, 137, 4424

Toomre, A. 1964, ApJ, 139, 1217

van der Hulst, J. M., Terlouw, J. P., Begeman, K. G., Zwitser, W., \& Roelfsema, P. R. 1992, in APS Conf. Ser. 25, Astronomical Data Analysis Software and Systems I, ed. D. M. Worrall, C. Biemesderfer, \& J. Barnes (San Francisco, CA: ASP), 131

van der Kruit, P. C., \& Searle, L. 1981, A\&A, 95, 105

Verheijen, M. A. W., \& Sancisi, R. 2001, A\&A, 370, 765

Wong, T., \& Blitz, L. 2002, ApJ, 569, 157

Yim, K., Wong, T., Howk, J. C., \& van der Hulst, J. M. 2011, AJ, 141, 48

Zschaechner, L. K., Rand, R. J., Heald, G. H., Gentile, G., \& Józsa, G. 2012 , ApJ, 760, 37 